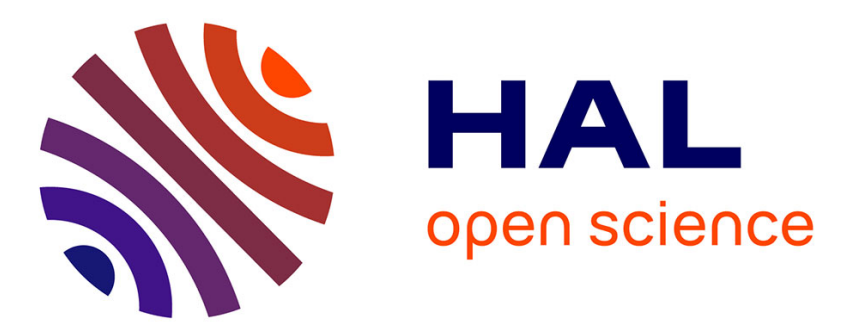

\title{
DZI: An Air Index for Spatial Queries in One-dimensional Channels
}

Kwangjin Park, Alexis Joly, Patrick Valduriez

\section{To cite this version:}

Kwangjin Park, Alexis Joly, Patrick Valduriez. DZI: An Air Index for Spatial Queries in One-dimensional Channels. Data and Knowledge Engineering, 2019, 124, pp.101748. 10.1016/j.datak.2019.101748 . lirmm-02386429

\section{HAL Id: lirmm-02386429 \\ https://hal-lirmm.ccsd.cnrs.fr/lirmm-02386429}

Submitted on 29 Nov 2019

HAL is a multi-disciplinary open access archive for the deposit and dissemination of scientific research documents, whether they are published or not. The documents may come from teaching and research institutions in France or abroad, or from public or private research centers.
L'archive ouverte pluridisciplinaire HAL, est destinée au dépôt et à la diffusion de documents scientifiques de niveau recherche, publiés ou non, émanant des établissements d'enseignement et de recherche français ou étrangers, des laboratoires publics ou privés. 


\title{
DZI: An Air Index for Spatial Queries in One-dimensional Channels
}

\author{
Kwangjin Park, Alexis Joly, and Patrick Valduriez ${ }^{1}$ \\ Wonkwang University, Korea, Inria, Montpellier, France, Inria, Montpellier, France
}

\begin{abstract}
The wireless data broadcast environment characteristics cause the data to be delivered sequentially via one-dimensional channels. A space-filling curve has been proposed for recent wireless data broadcast environments. However, air indexing introduces various problems, including the increase in the size of the index, conversion costs, and an increase in the search space because of an inefficient structure. In this paper, we propose a distribution-based Z-order air index and query processing algorithms suitable for a wireless data broadcast environment. The proposed index organizes the object identification (hereafter called ID) hierarchically only in terms of objects that are present. We compare the proposed technique with the well-known spatial indexing technique DSI by creating equations that represent the access time and tuning time, followed by conducting a simulation-based performance evaluation. The results from experimental show that our proposed index and algorithms support efficient query processing in both range queries and K-nearest neighbor queries.
\end{abstract}

Keywords: Moving objects, mobile computing, selective tuning, and spatial index.

\section{Introduction}

Most studies of Location-Based Services (LBS) technologies and services have been conducted with a server-centric approach. However, having all the work handled entirely by a server leads to problems such as high dependence of the client on the server, lack of consideration for moving objects, and privacy invasion [12][25-27]. Furthermore, query processing time can increase due to the overload of the server that processes individual clients' query requests. In particular, real-time information processing tasks for a large number of moving objects can put a heavy burden on the server [2] [9-11] [24]. Service delays due to server workloads in an environment where query targets and requesters move at the same time may result in wrong query results. To solve this problem, the workload of the server can be reduced by disallowing the server to access the client's individual queries.

In wireless data broadcast systems, popular information is periodically disseminated through one-dimensional communication channels (i.e. a channel in which information is sequentially delivered from a server) to mobile clients. The wireless data broadcast method is a one-to-many communication method that sends packets to the entire wireless network and is not affected by the number of clients [1-10][23][34][38]. In recent years, there 


\footnotetext{
${ }^{1}$ Spatial data is the data or information that identifies the geographic location of features and boundaries on earth, oceans etc.

${ }^{2}$ The average duration for getting to the next index segment is called probe wait $[6,7]$

${ }^{3} \mathrm{~A}$ space-filling curve is a continuous path that visits every space in a $k$-dimensional grid once without crossing itself.
} 


\footnotetext{
${ }^{4} \mathrm{~A}$ spatial index is a special access method used to retrieve spatial data from within the data-store.
}

caused by an unnecessary identifier can be reduced during index configuration. Moreover, our proposed index does not give location identifier information for a region where the object is not present. Therefore, information regarding moving objects can be efficiently managed, because the location of moving objects can be determined with a small amount of information.

The main contributions of this paper are as follows:

- We propose a lightweight spatial index that assigns a tree-based hierarchy ID (identification) only to objects that are present, and can manage the positions of moving objects. The main difference between our index and the conventional index, such as quadtree-based or grid-based index, is that the proposed index divides space and assigns bit-codes only to those regions where objects exist.

- We propose breadth-first and depth-first spatial query search algorithms whose application depends on the location distribution of the objects. The breadth-first provides excellent for tuning time especially when the object is evenly distributed. On the other hand, the depth-first provides excellent for access time by determining the final query result by listening to the partial data information.

- We propose a data delivery model that is in accordance with the location distribution of objects.

- We propose an algorithm for the continuous spatial query processing of moving objects that supports and facilitates the determination of the location of future objects, using the client's own calculation.

The remainder of this paper is organized as follows. Section 2 discusses related work. Section 3 discusses major performance factors and defines the system model. Section 4 describes the distribution-based Z-order index. Section 5 describes the data dissemination and selective tuning algorithms. Section 6 describes an efficient method for the location of moving objects. Section 7 evaluates the performance of distribution-based Z-order index with DSI and BBS. Finally, Section 8 concludes.

\section{Related Work}

First, we discuss the data broadcasting methods. Then, we discuss the spatial index ${ }^{4}$ techniques for the wireless data broadcast environment.

\subsection{Data Broadcasting}

The server transmits the information that the client wants by identifying that information in advance through a broadcast channel. For instance, advertisements, weather 
forecasts, traffic information, flight and train information, and other information can be regularly sent to unspecified individuals [31].

In [14], the authors introduce a technique for delivering data objects to the clients in asymmetric environments. In the environments, the network characteristics in downlink direction is faster than those in uplink direction. In this technique, groups of pages, such as hot and cold groups, with different broadcast frequencies are multiplexed on the same channel. Then, the items stored on the faster disks are broadcast more often than those items on the slower disks. In [19], the authors present the Broadcast on Demand (BoD) model to provide timely broadcast according to requests from users. The goal is to maximize performance with respect to satisfaction of deadline constraints and achieve efficient use of available bandwidth. In [20], the authors investigate how to efficiently generate the broadcast schedule in a wireless environment. They consider the access pattern formed by all the requests where the data dependency and access frequency can be represented by a weighted directed acyclic graph (DAG). In [21], the authors observe that in general, clients are divided into several groups, each one in a different location, with the members of each group having similar demands. Then, they propose a mechanism that exploits locality of demand in order to increase the performance of wireless data broadcast systems. In [22], the authors define two optimization problems, such as MCDR (Minimum Cost Data Retrieval) and LNDR (Largest Number Data Retrieval). MCDR aims at downloading a set of data items with the minimum energy consumption, whereas LNDR aims at downloading the largest number of requested data items in a given time interval. In this paper, authors consider data retrieval scheduling over multiple channels. In [32], authors address the problem of answering $K \mathrm{NN}$ ( $K$-Nearest Neighbor), range, and RNN (Reverse Nearest Neighbor) queries in road networks via broadcast channels. Then, they propose ISW (index for spatial queries in wireless broadcast environments)-index for spatial queries in wireless broadcast environments. The ISW provides a pair of distance bounds, which is effective for pruning the search space. The search space is reduced by using ISW and subsequently the client can download as less as possible data for query processing, which can conserve the energy. In [40], authors discuss the problem of spatial keyword query processing in road networks in wireless broadcast environments. Then they provide algorithms for various queries, such as boolean range, top- $k$ and ranked spatial keyword queries. In [33], authors address the problem of processing CNN (Continuous Nearest Neighbor) queries in road networks under wireless broadcast environments. Then, they propose a method to construct and partition the NVD (Network Voronoi Diagram) structure of the underlying road networks to derive an NVD quadtree and then transfer the tree into a linear sequence of data packets. In BBS[29], the data objects broadcast by the server are sequentially ordered based on their locations. Therefore, it is not necessary for the client to wait for the index segment if the desired data object is able to be identified before the associated index segment has arrived. In BBS, the structure of the broadcast affects the distribution of the data object. BBS provides the fast access time since no index is broadcast along with the data and, thus, the size of the entire broadcast cycle is minimized. The techniques discussed above focus on efficient data transfer by analyzing users' requests. However, most of them do not consider spatial queries, as we do in this paper. 


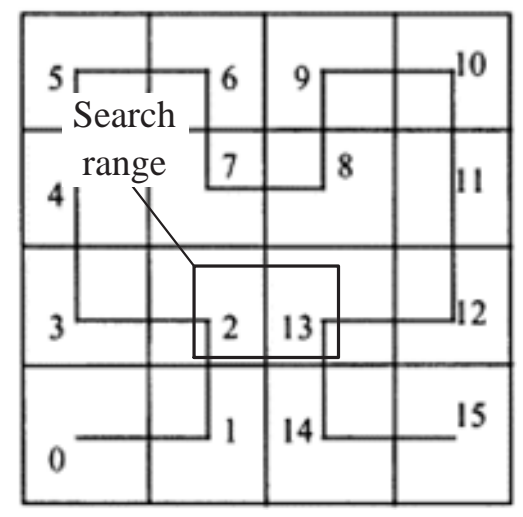

Figure 1: Access time with $\mathrm{HC}$ value for range query.

\subsection{Spatial Index on Air}

DSI has a distributed structure that mixes multiple search paths into a linear index structure that is distributed into the broadcast cycle. In DSI, a pointer of each data instance is repeated as many times as the number of entries of an index in a broadcast cycle to facilitate multiple search paths. Access time represents the period of time elapsed from the moment a user issues a query to the client to the moment when the required data item is received by the client. In each frame of the index, a pointer that is increased exponentially is stored; its role is to support fast access and selective tuning, i.e. mobile devices only selectively tune into broadcast channels when required. HC-based index techniques set up the value of the $\mathrm{HC}$ order based on the distribution of the object, and assign an identification number to every vertex of the basic curve, regardless of the presence or non-presence of the object. In this case, because the $\mathrm{HC}$ order does not represent a 1-1 mapping of the order of data delivery, an object mapping process is required for calculating the object position after confirmation of the $\mathrm{HC}$-value. In the process, the conversion time of $O\left(b^{2}\right)$ is consumed, with $b$ denotes the number of bits needed for the coordinates of the object. Also, in case the objects are located close to each other, the value of the HC of order, which is used to distinguish the position of the object, increases. As a result, many IDs are required, even though the total number of objects is not large. Furthermore, because of the nature of the $\mathrm{HC}$, problems may occur, depending on the location and scope of the query. An increased search space is needed in order to navigate through unnecessary blocks. For example, as can be seen in Figure 1, the client can obtain the final results only after waiting until objects 2 to 13 have been transmitted from the server for range query processing. The AT (access time) and TT (tuning time) will be increased further if the number of objects is to be increased and the query processing zones include more objects. In the $K \mathrm{NN}$ query processing, more serious problems will occur, depending on the value of ' $K$ '.

The problem with the HC-based distributed index can be summarized as follows:

- Even if a pointer is used that is exponentially increased by the exponential base $e$, when the number of the total data equals $N$, the number of the pointers will increase 
as much as $\log _{e} N$ in each index frame when $N$ increases. As a result, the size of the index will be increased, and the amount of information that needs to be listened to will increase significantly.

- Even if the existing index structures based on HC have hierarchical structures, in order to obtain the desired results, indexes should be sequentially read from the beginning of the exploration to its end. Tuning time may be reduced by proposing a distributed index structure consisting of overlapping pointers for selective tuning, but the increased index information lengthens the exploration time.

- In order for the object to confirm the location information, additional mapping, a conversion process for the $\mathrm{HC}$ value, and the location calculation of the object are required.

In [2], the authors propose an air indexing structure for supporting moving data objects. The goal is to reduce the power consumption and response time at the client side. They design algorithms for snapshot and continuous queries, over static or dynamic data with a regular grid. They apply other space-filling curves such as Peano curve for their index. However, this technique also presents the same structural problems (inefficient selective tuning) as the Hilbert-curve-based index. In [36], authors argued that bitmapbased indexing can be highly effective for running range query workloads on spatial data sets. Then, they introduced a compressed spatial hierarchical bitmap, called cSHB, index structure that takes a spatial hierarchy and uses that to create a hierarchy of compressed bitmaps to support spatial range queries. In [42], authors propose a multi-leveled air indexing scheme in non-flat data broadcasting (MLAIN) to process window queries with the popularity of spatial data items. MLAIN partitions the data space by recursively subdividing it into four quadrant cells of multiple levels until all cells satisfy the constraint that the number of data items in a cell is not over a specified number. In MLAIN, each cell having one or more hot data items, called a hot cell, is broadcast more frequently than regular cells to help clients quickly access hot data items on the channel. In [35], we proposed the Hierarchical Grid Index (HGI) to remedy the Hilbert-curve-based index. For the HGI, the location of the object is expressed by dividing grids until one grid contains one object. Because grid division and index ID distribution only occurs in the area with the object, the index pointer size can be reduced along with the index deciphering time and TT. The delivery order considers an object's location and delivers it using the grid recognizers' order to be awake, which occurs only when information close to the query point is received, and to process the final query results. However, to confirm the object's location information, coordinate information from the lowest level grid must be confirmed. [37] explores the problem of spatial query processing in road sensor networks by means of wireless data broadcast. The authors present a method to partition the record-keeping information about the underlying road sensor network and its associated objects, by a distributed air index, called integrated exponential index, based on an extended version of the Hilbert-curve. They also propose client-side algorithms to facilitate the processing of spatial queries. In [38], the authors propose a skewed spatial index considering clients' 
skewed access patterns in the non-uniform wireless broadcast environments. The index information considering the non-uniform broadcast is interleaved with the spatial objects on the wireless channel to support efficient access. In [39], we examine the problems experienced in the existing wireless broadcast environment and propose a novel system model and index technique. Instead of receiving spatial data information from the server, the client in the proposed system environment receives spatial data for part of the area from a sub_server to reduce the query processing time. In this manner, the model selectively tunes to the data located within the partial area most suitable for the query.

In parks or train stations, smartphone users may utilize location-based advertising messages to find information about nearby restaurants, hotels, and shops. If this makes their smartphone be flooded with a burst of advertising messages, they are likely to turn off advertising messaging services. In order to overcome the above problems, an index structure is required that gives the identification number by considering only the location of the objects actually present. In addition, fast and efficient algorithm for supporting spatial queries in wireless data broadcast environments should be developed.

\section{Preliminaries}

In this section, we examine the main problems affecting the performance evaluation. Then, we present the system model.

\subsection{Problem Statement}

In a wireless data broadcast environment, the increase of 'index size' and 'the irregular delivery order of data' increase AT and TT at the client side. First, let us consider the impact of the increase of index size on AT and TT. The conventional indexing schemes based on a space-filling curve, such as DSI allocates the index IDs to all zones even there is no object, thereby increasing the index size. This gives rise to the following problems:

- AT increases as the amount of data that is delivered during a single broadcast cycle increases.

- TT increases as the amount of data that needs to be read and processed by the client increases.

As shown in Figure 2, the pointer size directly influences the wireless data broadcast cycle. Figure 2(a) shows a single broadcast cycle in cases where overlapping pointers are not used and Figure 2(b) shows a single broadcast cycle in cases where overlapping pointers are used in the distributed index to reduce probe wait time. Let us assume that a logical time unit of both data object and index pointer be 1 . Let $N$ be the total number of objects. In distributed index, which uses overlapping pointers, the number of pointers increases by $\log _{e} N$ as $N$ increases [11]. Let the value of $N$ be 9 and $e$ be 2 . Then, each data object contains 4 pointers in distributed index. As a result, the total logical time of a single broadcast cycle for non overlapping pointers is 9 , whereas distributed index is 36 $(9 \times 4)$. Figure 2 shows an example which demonstrates the impact of adding the forwarding 


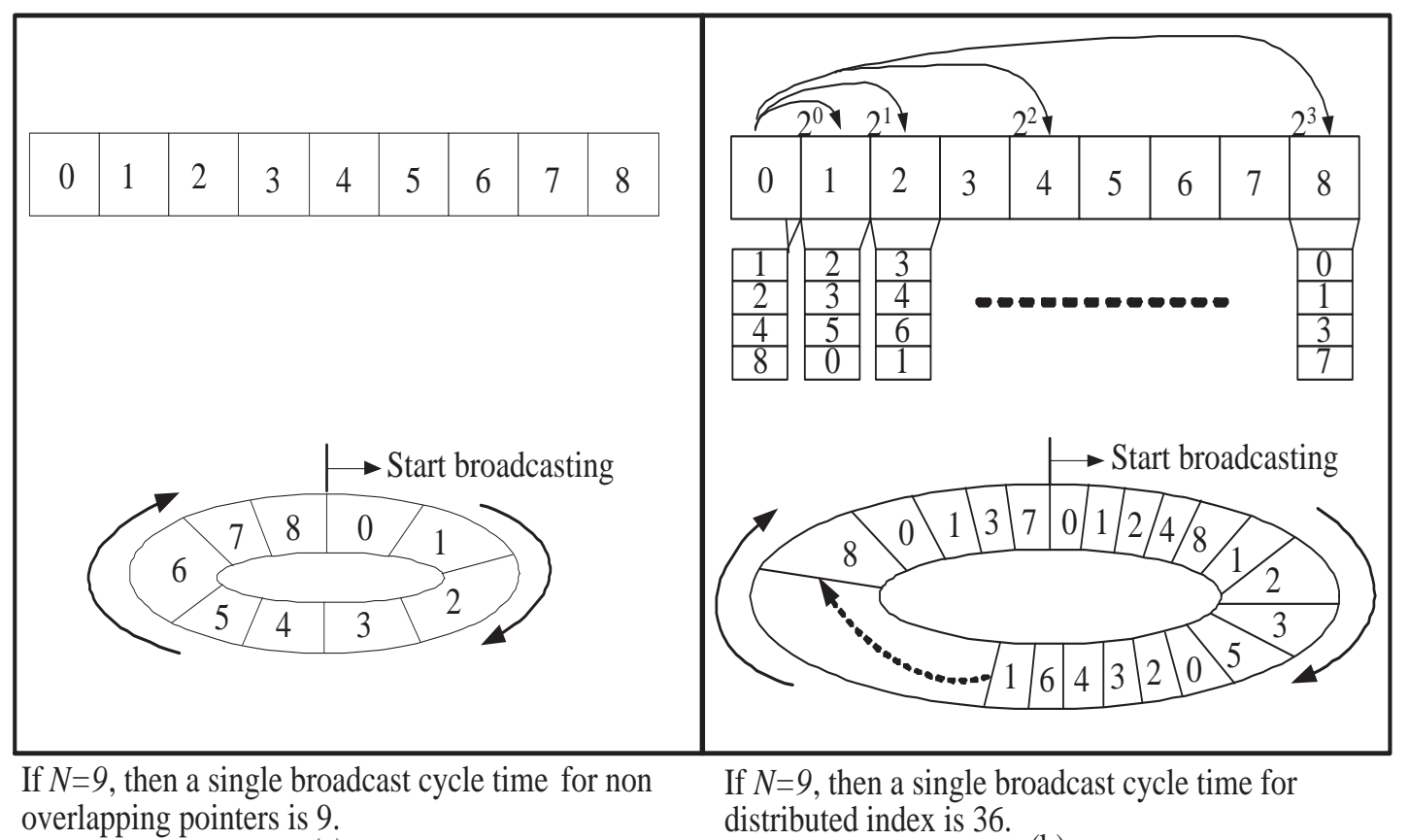

(a)

(b)

Figure 2: Access time according to the size of index pointers.

pointer to the existing spatial index on the broadcast cycle. The total logical time can be changed depending on the parameter value of $e$ and $N$ and the applying algorithms.

Second, let us consider the impact of the irregular delivery order of data on AT and TT. Let index_data be the size of an index that represents objects. If the delivery order ignores the location of the object, the query result can only be confirmed after the locations of all objects have been confirmed at the time of spatial query processing. Therefore, if the user data is delivered along with the index data, it becomes AT $=N \times$ index_data, $\mathrm{TT}=$ $N \times$ index_data. On the other hand, about half of the ordinary AT and TT are needed when data are delivered according to a certain predefined rule. Therefore, we get AT $=$ $\frac{N \times \text { index_data }}{2}, \mathrm{TT}=\frac{N \times \text { index_data }}{2}$.

We then define the problem as follows:

Input

- Locations of spatial objects

- Directions of spatial objects

- Velocities of spatial objects

Output

- Spatial index

Objective 
- Minimize index_data

- Minimize TT and AT

- Provide client's self-process for spatial query of a mobile object

In this paper, we aim to provide a lightweight spatial index that allows fast and efficient data access and tuning by delivering objects in a certain ordered fashion. In addition, the proposed index exploits the location, direction, and velocity of mobile objects to enable self-spatial query processing at the client.

\subsection{System Model}

Our system model can be summarized as follows:

- Server: The server receives reports regarding position, direction, and speed information from the moving object, and configures the index based on these reports. It then delivers the index, together with the user data, through the wireless data broadcast channel.

- Client: It is the user terminal that requests the service to a server. The client, which receives the user query, takes the following actions: (i) tries to listen to the index through the wireless data broadcast channel in wake-up mode; (ii) reads the index and is set to sleep mode after confirming all the information needed, and at what point it will be delivered; and (iii) wakes up selectively at the time of information delivery, and then tunes, after which it delivers the final result to the user.

- Objects: This refers to fixed objects (e.g., department stores, gas stations) or moving objects (e.g., people, taxis). These objects deliver information regarding their location, direction, and speed to a server in the jurisdiction area. In this paper, we assume that the points-of-interest belongs to the same types (e.g., gas stations, restaurants.) of queries.

- Data: Data can be classified into 'index data' and 'user data'. Index data includes the pointer for the object, its position, direction and speed, delivery time, etc. The index data includes the arrival time information of the adjacent index. If we assume that the client wakes up at random times, and if the first data that it listens to is the index data, it confirms the arrival time information of the adjacent index, and then waits again in sleep mode until the adjacent index arrives for power conservation. On the other hand, the user data represents specific information about the object (e.g., photos of goods, prices and maps in a department store).

Let $N$ be the total number of objects, $d$ be the user data size, and $p$ be the index pointer size.

- Channels: These are wireless data broadcast channels through which the server delivers the information, and the uplink channel through which the object conveys information regarding its location, direction, speed, etc. In this paper, we assume that spatial indexes and data be transmitted through a single wireless channel.

We assume that a server collects the locations of hotels, gas stations, and certain moving objects and manages them integrally. The client and object can identify their locations through the use of location measurement technologies such as GPS. The data 
Table 1: Definition of Notations.

\begin{tabular}{|c|c|}
\hline Notation & Description \\
\hline $\begin{array}{l}\text { query } \\
\text { point }\end{array}$ & The location where the query is issued \\
\hline K & The closest $K$ points to a query point \\
\hline bcast $n$ & A broadcast cycle $n$ \\
\hline $\mathrm{G}_{i}$ & A grid node $i$ which contains at least one object \\
\hline $\mathrm{O}_{i}$ & An object $i$ \\
\hline $\mathrm{QKNN}$ & $K$-nearest neighbor query \\
\hline$t$ & The time stamp (time interval) \\
\hline$N$ & The total number of data objects \\
\hline$e$ & the exponential base $(e>1)$ \\
\hline$w$ & The quantity of the objects that is kept in the queue \\
\hline$N^{\prime}$ & The tuned total number of data \\
\hline$f$ & The total index tuple size of the next pointers in each index table \\
\hline$h$ & The Hilbert-curve order \\
\hline$D$ & The size of data \\
\hline$T_{e 1}$ & The total size of the exponential pointer during a broadcast cycle \\
\hline$\Re$ & The number of objects that are contained in the grid nodes \\
\hline$p^{n}$ & The child node of the root grid node $\mathrm{R}_{n}$ \\
\hline$z^{n}$ & The number of grid nodes that belong to $\mathrm{R}_{n}$ \\
\hline GID & The grid identifier included in $\mathrm{R}_{n}$ \\
\hline$z^{\prime n}$ & The number of grid-nodes included in the $\mathrm{R}_{n}$ \\
\hline
\end{tabular}

that is delivered from the server is delivered at constant cycle intervals according to the rules given, and the client wakes up at random times.

Table 1 summarizes the notations used in this paper.

\section{Distribution-based Z-order Index}

The main performance factors we consider are AT and TT. These two factors are affected directly by the distribution and the number of objects, index size, and the order of delivery. We can summarize the conditions to be considered in this regard as follows.

- The client, without having to listen to the entire index data in order to handle spatial queries, can reduce AT.

- Having the client quickly determine the portion of the index information that does not need to be listened to can reduce TT. The client wakes up only when the needed data is delivered by identifying the part that is not a query target, and then tuning to the index data and user data. 


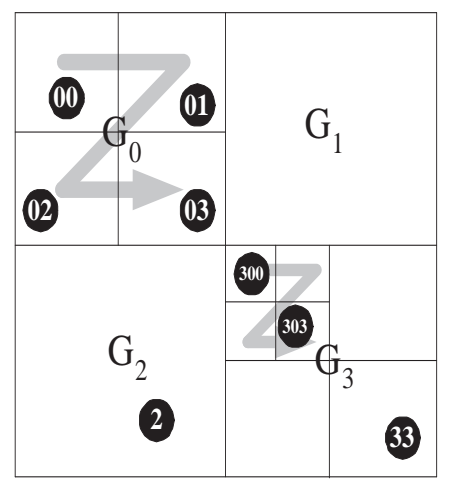

(a)

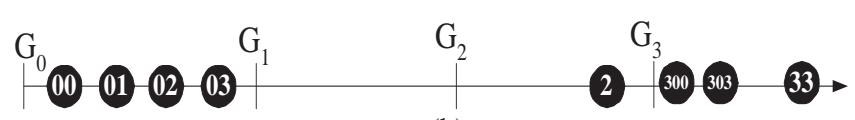

(b)

Figure 3: DZI index structure: (a) partitioning a region using a grid, (b) fit in one-dimensional environment.

- The reduction in the size of the index, which is composed of information regarding the object location and delivery time, can reduce AT and TT at the same time.

In order to satisfy these conditions, we can simplify the index data and user data to fit them in a one-dimensional environment.

In the proposed Distribution-based Z-order Index (hereafter called DZI), the index structure is determined on the basis of the location and distribution of the objects, and the server periodically rebuilds the index so that it reflects the mobility of the objects. DZI represents the locations of the objects in a grid structure. The order of delivery of an object is determined by the order of the numbers in the grid. The major different between DZI and Hilbert-curve-based index is that the Hilbert-curve-based index must allocate bitcodes to represent the whole regions regardless of whether there exists an object, whereas DZI divides space and assigns bit-codes only to those regions where objects exist. Thus, DZI supports to reduce the pointer size and search cost.

A grid number is assigned in the order of the character ' $\mathrm{Z}$ ' of the alphabet by dividing the total area into four divisions. Each number in the grid is used as the identification number that represents the location of the object.

DZI is based on the assumption that objects are delivered in order of ' $Z$ '. However, AT and TT are not influenced even if objects are delivered in other kinds of order-e.g., delivered in order of ' $\mathrm{N}$ '.

The basic structure of DZI is similar to a quadtree. However, the quadtree is an appropriate structure for a disk-based index, where the server receives and processes the clients' requests in an on-demand environment. On the other hand, DZI is an appropriate structure for an air-index, where the server transfers the broadcast programming information and the actual broadcast contents in sequence via wireless broadcast channels and the clients use the received index data to selectively tune to the necessary broadcast [35]. That is, 


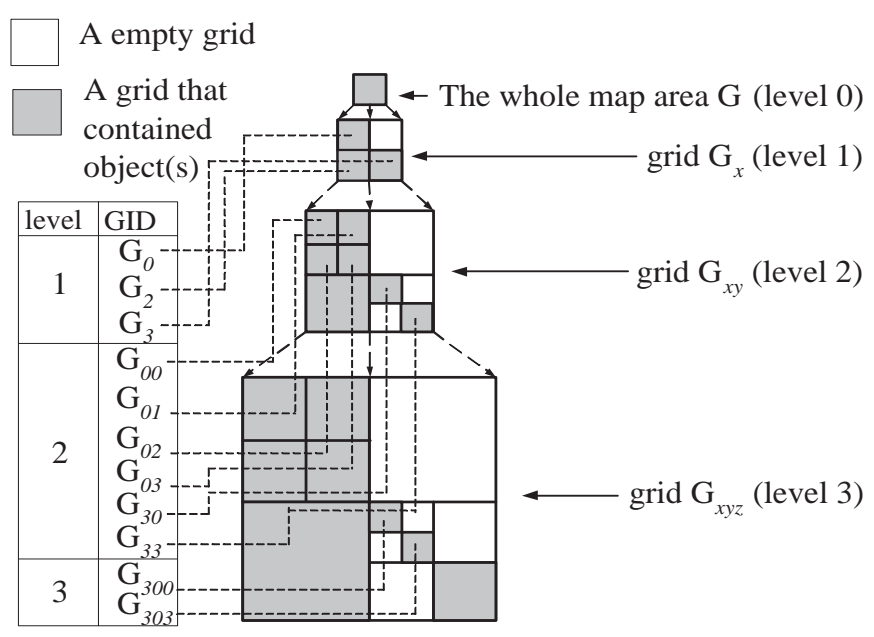

Figure 4: DZI hierarchy and its ID.

DZI provides ID only to objects that are present that enables more powerful accesses with a lightweight spatial index that allows fast and efficient data access and tuning.

We note that it is not necessary to wait until the beginning of the next broadcast cycle even though a client loses some data since grid ID represents its location similar to the point number of HC-based index [13]. In the DZI, the partitioning process varies according to the relative locations and distribution among the objects (see Figure 3(a)). The biggest difference between the existing HC-based index and a grid-based index is that the identification number of the DZI is granted only to objects that are present as targets. The search costs for index, mapping, and conversion can be reduced by reducing the amount of unnecessary ID information. This actually avoids having pointers on empty regions and reduces the index size. Then, the client can confirm an object's location using only its grid identification number, which has a hierarchical structure; in this way, the client is able to perform selective tuning. Another feature of DZI is that the index pruning ability is enhanced by means of a vertical hierarchy at the time of index browsing. The division of the grid repeats the division into four parts, depending upon the distribution or location of an object, and divides the parent nodes (top grid) into child nodes (sub-grid) until it contains only the last single object. In this scheme, the parent node becomes $G_{0}$, $G_{1}, G_{2}, G_{3}$, after which the four nodes will again have child nodes. For example, let us assume that the $G_{0}$ node has objects in all four regions; they will then have child nodes of $G_{00}, G_{01}, G_{02}, G_{03}$. Let us assume that the $G_{00}$ node has all the objects in the four division regions; they will then again have child nodes with identification numbers of $G_{000}$, $G_{001}, G_{002}$, and $G_{003}$. Consider Figure 4 and the object status on the $G_{0}, G_{1}, G_{2}, G_{3}$ grid:

- $G_{0}$ : only one object is present in all four grids, including $G_{00}, G_{01}, G_{02}$, and $G_{03}$.

- $G_{1}$ : none of objects is present.

- $G_{2}$ : a single object is present. 
- $G_{3}$ : one of each object is present in three different grids: $G_{300}, G_{303}$, and $G_{33}$.

This object status is expressed in one-dimensional structures shown in Figure 3(b). The structure of the tree is affected by the distribution of the objects. For example, a child node is not blindly divided into four grids. Rather, if the objects are distributed evenly, the tree will have parallel balance, and if the objects are located in a particular area of focus, they will exhibit an imbalanced appearance. Figure 4 shows the DZI hierarchy and ID. As the lower down object goes in the lower levels, the GID (Grid ID) number increases. The client can read the GID number and the number of digits and infer the search area and the range.

DZI is composed of root, intermediate and leaf entries. We can now define the parent and leaf of the DZI as follows.

Definition 1. (DZI Parent) Parent's grid ID represents the location of the grid on a map. The parent node contains a pointer of the child node(s) that represents the delivery time of each child node. The parent node has four child nodes and above two child nodes of the parent node have an object or at least one child node of the parent node has above two objects. That is, the division process only occurs to the grid node which has above two objects.

Definition 2. (DZI Leaf) The leaf node contains a pointer of the object (user data) which represents the delivery time of each object.

The DZI division satisfies the following property:

Property 1. (DZI division) In a given set of data regions, a grid $G_{i}$ repeats the hierarchy division process until it contains only one object. A parent grid can have four or none immediate child grids, and a child grid will have one parent grid.

The number of objects contained in a grid $G_{i}$ in the DZI satisfies the following property:

Property 2. (Number of objects inside $G_{i}$ ) Let num_obj_G $G_{i}$ be the number of objects inside $G_{i}$, which has child nodes. Let num_obj_ $G_{i}^{\prime}$ be the number of objects inside $G_{i}$ ', which has no child node. Then, num_obj_G ${ }_{i} \geq 1$ and num_obj_G ${ }_{i}^{\prime} \geq 0$.

The main components of an index segment are: the next broadcast start time; the first delivery data arrival time, according to the level (or grid ID) number; user data arrival time, object's $x$ - $y$ coordinates, and the number of objects within the grid. Figure 5 shows the detailed structure of the index data for one complete broadcast cycle. At the beginning of the index, it contains information that indicates the starting time of each block. We assume that the delivery order of object $O_{i}$ is delivered sequentially according to the grid numbers. 


\begin{tabular}{|c|c|c|c|c|c|c|}
$\begin{array}{c}\text { Information indicating } \\
\text { the starting time of each block }\end{array}$ & $\begin{array}{c}\text { Next broadcast } \\
\text { cycle time }\end{array}$ & $\begin{array}{c}\text { Arrival time for the first data } \\
\text { item of each level (or grid id) }\end{array}$ & $\begin{array}{c}\text { Arrival time } \\
\text { for user data }\end{array}$ & $\begin{array}{c}\text { Grid } \\
\text { IDs }\end{array}$ & $\begin{array}{c}\text { Number of objects } \\
\text { inside grid }_{i}\end{array}$ & $\begin{array}{c}\text { Details of } \\
\text { object }\end{array}$ \\
\hline
\end{tabular}

Figure 5: A detailed structure of the index data.

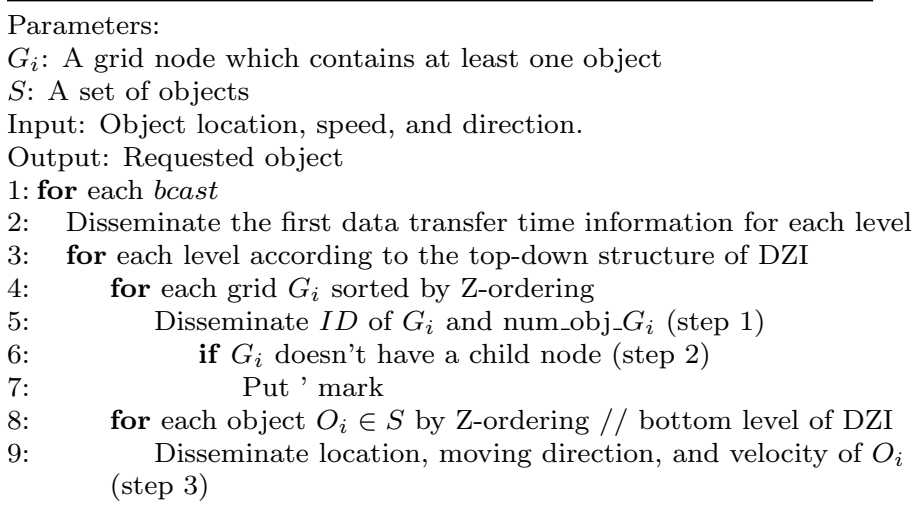

Figure 6: Data Dissemination algorithm for BFD.

\section{Data Dissemination and Selective Tuning on Air}

The server configures DZI in the form of a snapshot, based on the locations of objects within the service region, which are delivered periodically. The lowest level of the index in the configuration of a hierarchical grid node includes information regarding an object, i.e., its coordinates, speed of movement, and direction. The information regarding an object is updated and delivered in each cycle. In the DZI at the time of spatial query processing, because the objects are delivered from the server after being divided into a particular order sequentially and are hierarchically based on the location, they can selectively tune to only the objects in the region that they want to listen to. Our method supports selective tuning to the index with reference to each object's position, orientation, and maximum speed, not only in the case of a selective index tuning to static objects but also in the case of continuous spatial query processing of moving objects. To support efficient query processing, we divide our delivery method into BFD (breadth-first-delivery) and DFD (depth-first-delivery).

\subsection{Breadth-First-Delivery}

BFD delivers nodes in order from the top level of the index tree to the lower levels. BFD progresses by delivering the first root node of the DZI that appears and thus going deeper and deeper until a leaf node is delivered. The algorithm of BFD is shown in Figure 6. Please refer the following steps. The server in each level unit delivers the ID of $G_{i}$ and the number of objects inside of $G_{i}$ (step 1). If $G_{i}$ no longer contains a child node, it inserts an apostrophe (') and informs that the grid is not divided any further (step 2). At the lowest level, the details of an object, i.e., its $x-y$ coordinates, speed, and direction, are delivered (step 3). The information regarding how many child nodes the top node has, and the number of contained objects, can be obtained and considered at the time of $K \mathrm{NN}$ query 


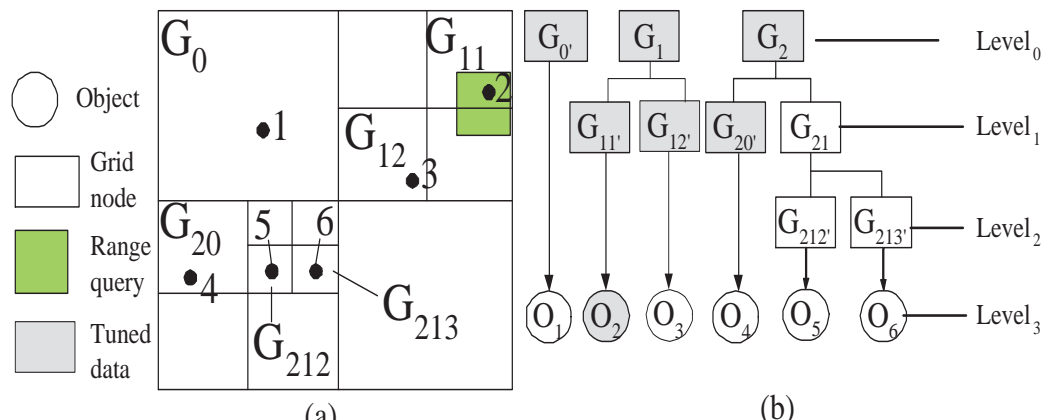

(a)

(b)

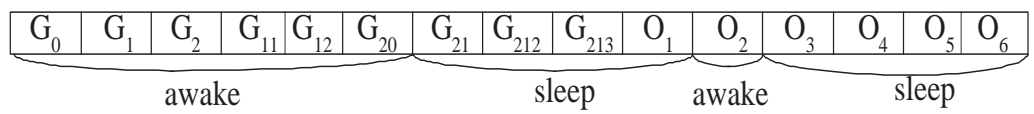

(c)

Figure 7: Breadth-First-Delivery: (a) objects and hierarchical grid division. (b) grid tree structure. (c) data listening.

processing. Because BFD has to listen to the data at the lowest level for the last query processing, the efficiency of AT is lower. However, it is possible to listen selectively by pruning the part that may not need to listen to by checking the top node first. Therefore, BFD provides the efficiency of TT; in particular, the object is evenly distributed and the efficiency of the search is excellent. We note that, in BFD, AT might decrease when the object data and the index data are delivered at the same level. However, in this case, additional information regarding the arrival time of object and index data deliveries at each level is required, which can lead to increase both AT and TT. Thus, our indexing scheme assumes that there is a distinction between the object data delivery and the index data delivery in BFD.

In Figure 7, let us look at the process of spatial query processing that uses BFD. Let us assume the range query for ease of understanding. In Figure $7(\mathrm{a}), G_{1}$ is included in the query range (a possible object for the answer of range query). The grid and the circle indicated in light gray denote the data that the client needs to listen to. After listening to level 0 , the client finds that there are two objects in $G_{1}$ (see Figures $7(\mathrm{a})$ and $7(\mathrm{~b})$ ). After verifying up to the $G_{20}$ at level 1, the client can find each of $G_{11}$ and $G_{12}$ in $G_{1}$ with an object within. The query range is set to include $G_{11}$ and $G_{13}$, and no object is present in $G_{13}$. The client is switched to sleep mode after listening as far as $G_{20}$ in level 1, and wakes up at the time of object delivery of level 3. It then obtains the object for $\mathrm{O}_{2}$, and returns the final query result. In Figure 7(c), when a logical time unit is denoted by 1, TT is 7 , and AT is 11.

Table 2 shows the order of delivery according to the level of BFD. The client's KNN algorithm for BFD (see Figure 8) is as follows (the range query algorithm is omitted because it is similar and simpler to that of $K \mathrm{NN}$ ). Please refer the following steps.

The client confirms the number of objects that are included in the ID of nodes $G_{i}$ and $G_{i}$ for each level units (leaf nodes are excluded) (step 1-step 3). If $G_{i}$ belongs to the $K \mathrm{NN}$ query range (a possible object for the answer of $K \mathrm{NN}$ query) when the query point and 
Table 2: The order of delivery of the index data and the user data according to level of BFD.

\begin{tabular}{||l||l|}
\hline Level No. & data \\
\hline 0 & $G_{0}\left(1^{\prime}\right), G_{1}(2), G_{2}(3)$ \\
\hline 1 & $G_{11}\left(1^{\prime}\right), G_{12}\left(1^{\prime}\right), G_{20}(1)$, \\
& $G_{21}(2)$ \\
\hline 2 & $G_{212}\left(1^{\prime}\right), G_{213}\left(1^{\prime}\right)$ \\
\hline 3 & $O_{1}, O_{2}, O_{3}, O_{4}, O_{5}, O_{6}$ \\
\hline
\end{tabular}

Parameters:

$K N^{\prime}$ ': $K$ NN candidate

Input: Index

Output: Requested object

1: for each bcast

2: for each level except leaf level (step 1)

3: $\quad$ for each grid $G_{i}$ in Z-order (step 2)

4: $\quad$ read $n u m_{-}$obj_G $G_{i}$ (step 3)

5: $\quad$ check whether $G_{i}$ is located inside $K \mathrm{NN}^{\prime}$ ' range or not

6: $\quad$ if $G_{n}$ is located inside $K \mathrm{NN}$ range

7: $\quad K \mathrm{NN}^{\prime} \leftarrow K \mathrm{NN}^{\prime} \cup G_{n}$ (step 4)

8: $\quad$ else $K \mathrm{NN}^{\prime}=K \mathrm{NN}^{\prime}-G_{n}$ (step 5$)$

09: for each object $O_{i} \in G_{n}$ from leaf level, where $G_{n} \in K \mathrm{NN}$

10: $\quad$ check whether $O_{i}$ is located inside $K N N$ range or not

11: if $O_{i}$ is located inside $K \mathrm{NN}$ range

12: $\quad K \mathrm{NN}^{\prime} \leftarrow K \mathrm{NN}^{\prime} \cup O_{i}$

13: $\quad$ else $K \mathrm{NN}^{\prime}=K \mathrm{NN}^{\prime}-O_{i}$

14 return $K \mathrm{NN}$ objects, where object $\in K \mathrm{NN}^{\prime}$ (step 6)

Figure 8: $K \mathrm{NN}$ algorithm for BFD. 


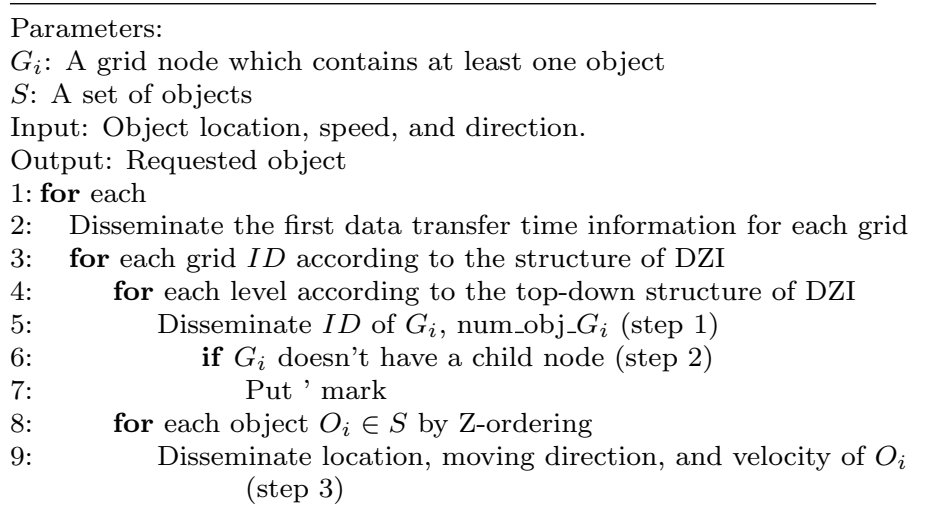

Figure 9: Data Dissemination algorithm for DFD.

the distance of $G_{i}$ are compared, it is included in the $K \mathrm{NN}$ candidate set (step 4). If $G_{i}$ does not belong to the $K \mathrm{NN}$ query range, it is excluded from the $K \mathrm{NN}$ candidate set and the next $G_{i}$ is checked. The client reads the object on the inside of $G_{i}$ that is contained in the $K \mathrm{NN}$ candidate set at the level of the last leaf, and returns the final $K \mathrm{NN}$ result (step 5). It is important to notice that in the search process, $G_{i}$ that belongs to $K \mathrm{NN}$ can be changed continuously. In other words, the most distant $G_{i}$ that has previously been an element of $K \mathrm{NN}$ is excluded from $K \mathrm{NN}$ when a new $G_{i}$ is included.

\subsection{Depth-First-Delivery}

The following DFD technique delivers information that belongs to the same grid, first. The components of the index and the delivery order consist of the number of each grid and delivery time, number of objects inside a grid, and object information (i.e., location, speed, direction, etc.). The algorithm of DFD (see Figure 9) is as follows. For each grid ID unit, the server delivers the number of objects that are included in the ID and $G_{i}$ for node $G_{i}$, which contains the objects (step 1 ). If $G_{i}$ no longer contains child nodes, it informs the client that the grid is not being divided any further by inserting an apostrophe (') (step 2 ). DFD delivers details about the object that belongs to the end $G_{i}$, which is not divided further according to Z-ordering, the $x-y$ coordinates of the object, speed and direction, etc. (step 3). Because the division node and object information are immediately delivered by the grid at the time of spatial query processing, query processing can be performed without having to wait unconditionally until the lowest level, as in BFD. Therefore, compared to $\mathrm{BFD}$, AT gets much better. In particular, the performance gets better when the distribution of objects is clustered in a particular area. Note that we assume that the index information is delivered by a certain $t$ time interval. Therefore, it is possible for the client to predict when the desired index information arrives, because of the nature of the index, which is delivered sequentially according to the order of the location at an interval of $t$ hours.

Let us consider the range query processing that uses DFD, keeping Figures 10(a) and 10 (b) in mind. In the figure, the query range is shown to be located within $G_{1}$. The grid 


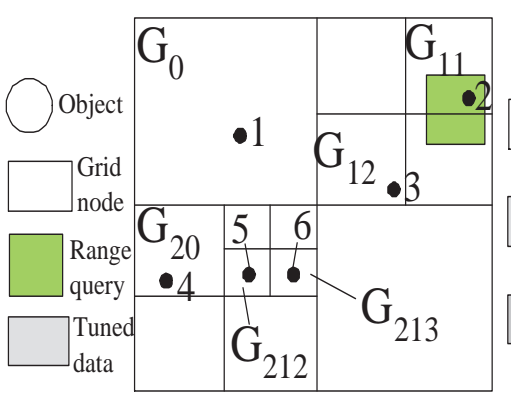

(a)

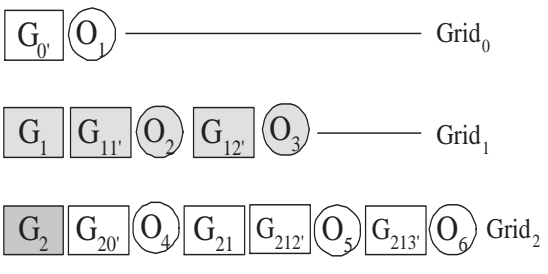

(b)

\begin{tabular}{|c|c|c|c|c|c|c|c|c|c|c|c|c|c|c|}
\hline $\mathrm{G}_{0}$ & $\mathrm{O}_{1}$ & $\mathrm{G}_{1}$ & $\mathrm{G}_{11}$ & $\mathrm{O}_{2}$ & $\mathrm{G}_{12}$ & $\mathrm{O}_{3}$ & $\mathrm{G}_{2}$ & $\mathrm{G}_{20}$ & $\mathrm{O}_{4}$ & $\mathrm{G}_{21}$ & $\mathrm{G}_{212}$ & $\mathrm{O}_{5}$ & $\mathrm{G}_{213}$ & $\mathrm{O}_{6}$ \\
sleep & \multicolumn{1}{c|}{ awake }
\end{tabular}

(c)

Figure 10: Depth-First-Delivery: (a) objects and hierarchical grid division. (b) grid tree structure. (c) data listening.

Table 3: The order of delivery of the index data and the user data according to GID.

\begin{tabular}{||l||l|}
\hline Level No. & data \\
\hline 0 & $G_{0}\left(1^{\prime}\right), O_{1}$ \\
\hline 1 & $G_{1}(2), G_{11}\left(1^{\prime}\right), O_{2}, G_{12}\left(1^{\prime}\right)$, \\
& $O_{3}$ \\
\hline 2 & $G_{2}(3), G_{20}\left(1^{\prime}\right), O_{4}, G_{21}(2)$, \\
& $G_{212}\left(1^{\prime}\right), O_{5}, G_{213}\left(1^{\prime}\right), O_{6}$ \\
\hline 3 & null \\
\hline
\end{tabular}

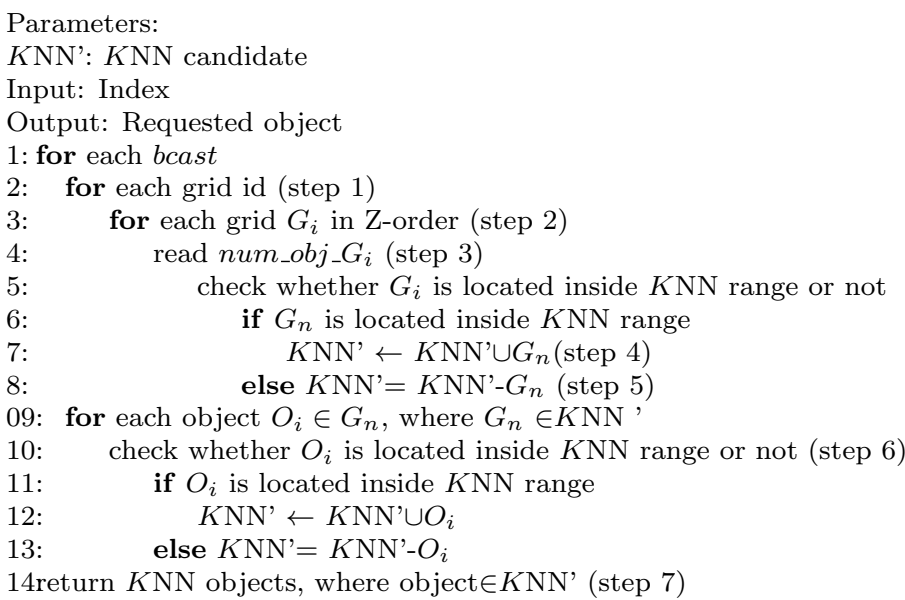

Figure 11: $K \mathrm{NN}$ algorithm for client DFD. 
and circle in light gray indicate the data that the client needs to listen to. The client checks the first data delivery time information of each grid. Next, the client wakes up at the $G_{1}$-level and listens to the data up to $G_{2}(3)$ in level $G_{2}$, then finally checks to confirm that objects $O_{2}$ and $O_{3}$ are in grid1, and that the object that belongs to the range query is $\mathrm{O}_{2}$. In 10(c), when one unit of logical time is denoted as 1 , TT is 6 , which is the wake-up time, and AT is 8, which is the sleep time + wake-up time. Selective tuning is possible using the grid ID in the case of DFD. In this case, the grid that is the query result target must be tuned to entirely until it finds the desired object. Thus, the amount of information that the client must tune to in its waking state is increased according to the distribution and the number of objects. In this case, more tuning time can be consumed than BFD. Table 3 shows the order of delivery of the index data and the user data according to GID.

The client's KNN algorithm for DFD (see Figure 11) is as follows (the range query algorithm is omitted because it is similar to that of $K \mathrm{NN}$ ). The client checks the ID of node $G_{i}$ and the number of objects that are included in $G_{i}$ by each grid ID unit (step 1 - step 3). If $G_{i}$ belongs to the $K \mathrm{NN}$ query range, which is determined by comparing the distance between the query point and $G_{i}$, it is included in the $K \mathrm{NN}$ candidate set (step 4). If $G_{i}$ is outside the scope of the $K \mathrm{NN}$ query range, it is excluded from the $K \mathrm{NN}$ candidate set, and then the next $G_{i}$ is checked (step 5 ). The client reads the objects within $G_{i}$ that are included in the $K \mathrm{NN}$ candidate set, and configures the $K \mathrm{NN}$ candidate set by checking if it is included in the $K \mathrm{NN}$ query range (step 6). It then returns the final results of $K \mathrm{NN}$ (step 7).

In summary, BFD, which searches through the indexes at all levels, has a longer access time than does DFD, which locates desired data objects via partial index information. However, BFD yields better tuning time because it exploits high-level index information to prune unnecessary search ranges beforehand. By contrast, DFD examines every data object in each partitioned zone via a depth-first search, making its tuning time longer than that of BFD. The server delivers the object location information periodically, using the information regarding the object's location, direction, and speed. The client performs spatial query processing by taking advantage of the object information that was delivered from the server. The client wipes off the objects that are not taken into account by using the information on location, direction, speed, and stores, etc., and manages only those objects that will affect future query results. Therefore, this process can reduce the computation time for updating and can reduce the dependence on the server. If the speed or direction of an object exceeds the predicted scope within the range and time where it is desired to be looked for, the server data of the next cycle must be tuned to.

When search ranges for $K \mathrm{NN}$ queries are updated by the client, the BFD and DFD algorithms utilize the following two principles. First, when the $K$ in $K \mathrm{NN}$ queries is decreased, the existing query processing results are used without the need to listen to the broadcast server. Second, when the $K$ in $K N N$ queries is increased, query processing is repeated by once again listening to the servers broadcast channel. 

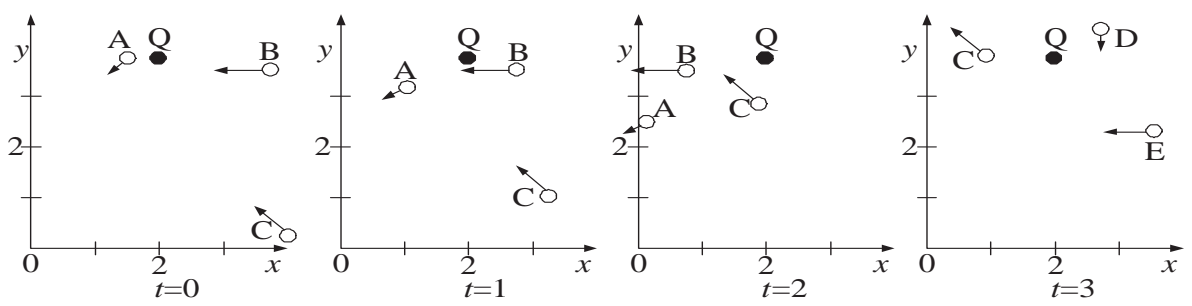

Figure 12: $3 \mathrm{NN}$ objects for a period of time $T_{0}-T_{3}$.

\section{Client's Own Calculation for KNN Queries on Moving Objects}

In this section, we examine a client's method to self-process a spatial query of a mobile object. For ease of presentation, we describe the query processing method focusing on snapshot KNN queries over static query point and dynamic objects. The client's query processing can be summarized as follows:

- The client performs its own future spatial query processing without having to rely on a server by receiving the information from the server regarding the object's location, speed, and direction of movement.

- The quantity of objects that is kept in the client's queue $(w)$, and the objects that are at a greater distance than the information stored in the queue among the information transmitted from the server, are excluded from the $K \mathrm{NN}$ target.

- The point in time at which the time stamp $t$ is changed is the point in time at which the target of $K \mathrm{NN}$ or the priority can be changed.

Figure 12 shows an example with $K=3(3-\mathrm{NN})$ in the client's $K$ NN query processing. In the figure, let us assume that object $\mathrm{A}$ and object $\mathrm{D}$ move at 0.5 increments of the maximum travel distance (with maximum speed) for the unit time $t$, and that object $\mathrm{B}$, object $\mathrm{C}$, and object $\mathrm{E}$ move at increments of 1 (with maximum speed). As can be seen in the figure, $3-\mathrm{NN}$ is processed up to $T_{0}-T_{3}$ using the object's information received from the server in the first cycle. In $T_{3}$, new objects $\mathrm{D}$ and $\mathrm{E}$ have been added, with the object information received from the server in the second cycle, and objects B and A are excluded from the $K \mathrm{NN}$ results target. Table 4 shows the 1 st NN, 2nd NN, and 3rd NN resulting objects according to $T_{n}$. The table shows that the client performs its own spatial query processing without having to rely on a server for a period of time, by using an index that has been delivered through a wireless data broadcast channel. The client is then passed through a wireless data broadcast channel using an index for a period of time, in order to perform its own query processing of spatial information. Let us assume that objects travel over a period of time in a straight line, like cars on the highway. The client's query processing can then be explained using the example in Figure 13. Observe the range queries and 3-NN processing by dividing the query processing time into logical time units of up to $T_{0}-T_{3}$. Assume that the server's broadcasting of object information of up to 1-4 to bcast 1 
Table 4: $3 \mathrm{NN}$ resulting objects according to $T_{n}$

\begin{tabular}{|c|c|c|c|}
\hline Time stamp & 1st NN & 2nd NN & 3rd NN \\
\hline$T_{0}$ & $\mathrm{~A}$ & $\mathrm{~B}$ & $\mathrm{C}$ \\
\hline$T_{1}$ & $\mathrm{~B}$ & $\mathrm{~A}$ & $\mathrm{C}$ \\
\hline$T_{2}$ & $\mathrm{C}$ & $\mathrm{B}$ & $\mathrm{A}$ \\
\hline$T_{3}$ & $\mathrm{D}$ & $\mathrm{C}$ & $\mathrm{E}$ \\
\hline
\end{tabular}

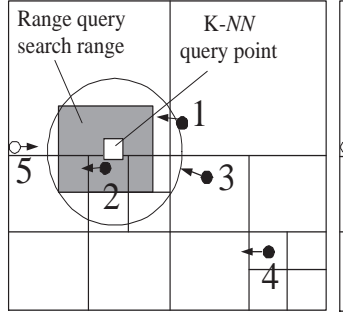

(a) $\mathrm{T}_{0} \sim \mathrm{T}_{1}$

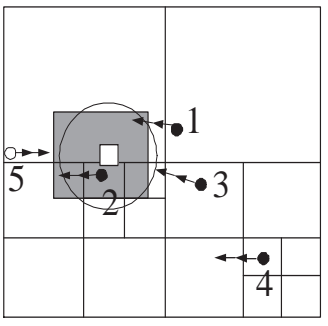

(b) $\mathrm{T}_{2}$

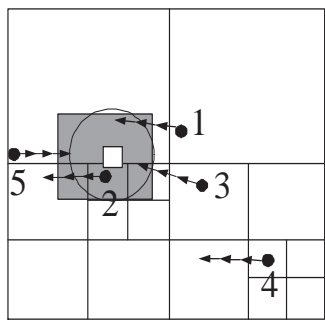

(c) $\mathrm{T}_{3}$

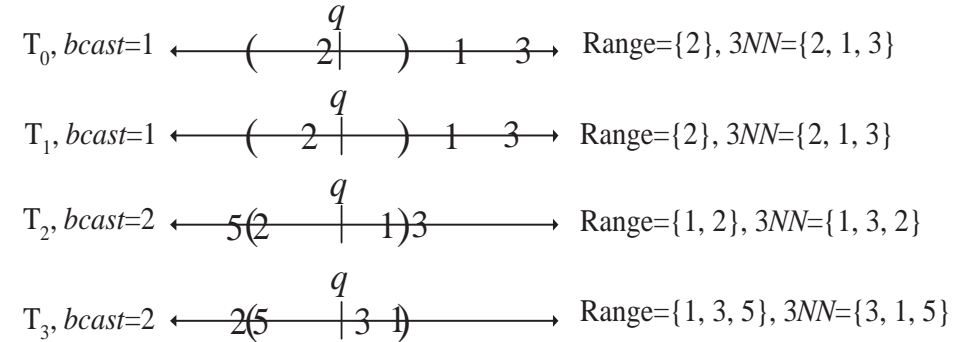

(d)

Figure 13: 3NN query processing for moving objects.

is the first broadcast cycle, and that it broadcasts the object information of 1-5, including new object 5 , to bcast 2 . In Figure 13, the empty box represents the query point in the $K N N$ query; the gray box, the range query; the black circles and arrows (e.g., $O_{1}, O_{2}, O_{3}, O_{4}$ ), the objects that have been confirmed for the location and direction from the server; and the empty circles with arrows (e.g., $O_{5}$ ), objects that have not yet been confirmed by the server. The objects such as cars, trains, bikes, and boats have the maximal travel speed. For example, a moving car may not exceed a speed of $300 \mathrm{~km} / \mathrm{h}$. An arrow represents the travel direction of an object, and the length of a line represents the maximum travel speed of an object, which increases by one scale every time $T_{n}$ increases. The client can predict the object's location in advance at the time of $T_{n}+n$, with reference to the object's current location, movement speed, and direction of travel, since the logical time $T_{n}$. In Figure $13(\mathrm{~d})$, the parentheses in a range of segments indicate the range query region, and the numbers in parentheses in the query results for $3 \mathrm{NN}$ are listed in order of the closest object. We assume that the client's queue can store a maximum of three objects, using $w$ $=3$.

Let us consider the snapshot of $K \mathrm{NN}$ query processing at the time $T_{0}-T_{3}$, considering the movement of the object. The $K \mathrm{NN}$ algorithm is as follows (the range query algorithm 


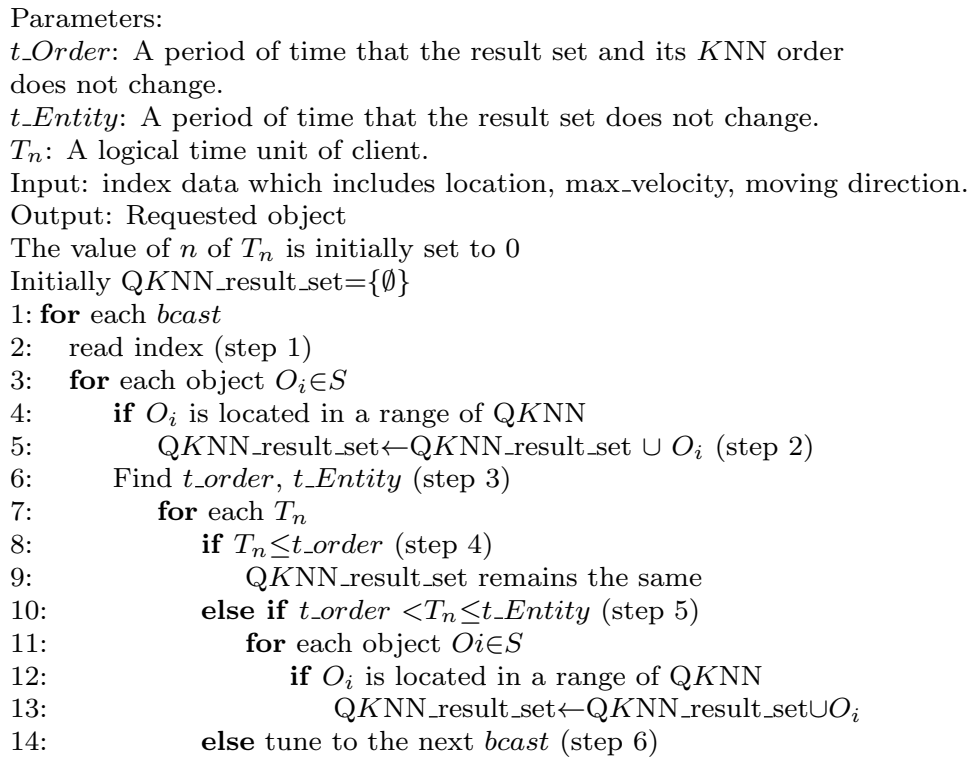

Figure 14: KNN algorithm for snapshot query.

is omitted because it is similar to that of $K \mathrm{NN}$ ).

Let us now explain the $K \mathrm{NN}$ algorithm by applying it to the example in Figure 14 . The client listens to the index received from the server (step 1). If the location of the object is included in $3 \mathrm{NN}$, it is included in Q3NN_result_set (step 2). The client then looks for $t_{-}$Order and t_Entity with reference to the object's location, direction of movement, and speed, which have been listened to (step 3). The index that has been delivered by the server in bcast 1 is then read, and the Q3NN_result_set $=\left\{O_{2}, O_{1}, O_{3}\right\}$, which represents the query results from $T_{0}-T_{1}$, ensures that it does not change (step 4 ). Thus, up to $T_{0}-T_{1}$ can obtain the same $3 \mathrm{NN}$ query result without listening to the server's index information or confirming the distance between the query point and the object. In addition, the order of the query results in $T_{2}$ is changed to $Q 3 N N_{\text {rresult_set }}=\left\{O_{1}, O_{3}, O_{2}\right\}$, but we can see that there is no object that can be included in the query result apart from objects $O_{1}, O_{2}$, $\mathrm{O}_{3}, \mathrm{O}_{4}$, which have been listened to in bcast1 (step 5).

The reason for this is that $O_{5}$ is not included in the $3 \mathrm{NN}$ query results by $T_{2}$, which have not been listened to in bcast1. In Figure 13, we can see that the target of $K \mathrm{NN}$ and the order of $K \mathrm{NN}$ at up to $T_{0}-T_{1}$ do not change. Thus, the client can return the stored value in the query result queue to the same $K \mathrm{NN}$ query result without the need for a separate comparison calculation during the period $T_{0}-T_{1}$. We can also see that the target has not been changed, even though the order of the objects for the $K \mathrm{NN}$ query result have been changed at up to $T_{0}-T_{2}$. Thus, from $T_{0}-T_{2}$, the client can obtain $K N N$ query results by comparing the object's location, direction of movement, and speed, which have been stored in the queue, without having to rely on the broadcast data of the server. After $T_{3}$, the client listens to the bcast 2 information that is delivered by the server, because a new object (see $O_{5}$ in Figure 13(c)) can affect the 3NN query processing results (step 6). It checks 
the information regarding the new $O_{5}$ using bcast2 information. In $T_{3}$, the query result of Q3NN_result_set $=\left\{O_{3}, O_{1}, O_{5}\right\}$ is obtained by additionally reflecting the information on the location, speed, and direction of $O_{5}$ to the range query and $3 \mathrm{NN}$. Let $t_{-} O$ Order be the time range from the present time until the query result target object and the $K \mathrm{NN}$ order are not changed $\left(T_{0}-T_{1}\right.$ of Figure 13), and $t_{-}$Entity be the time range from the present time until the query result target object is changed $\left(T_{0}-T_{2}\right.$ of Figure 13$)$. Thus, the client maintains information about objects as much as is allowed within the range of the queue, and is able to process spatial queries on its own by using $t_{-} E n t i t y$ and $t_{-} O r d e r$, without having to rely on the broadcast data of the server over a period of time.

In order to support client's self-process for spatial query of a mobile object, we propose two heuristics, each defining the unchangeable time period in which the target object of $K \mathrm{NN}$ does not change. Let us also assume that (1) objects that are present may disappear and new objects may be added in $M$, which denotes all service regions, in every bcast of the server, and (2) the server delivers the information regarding all present objects in $M$ in each cycle.

Heuristic 1. (The unchangeable time period in which the target object of KNN does not change). Let us assume that the time point that has received the object via the most recent bcast is Tlatest, and that any future time point is Tfuture. Let $A$ be the set of objects included in $K N N$ at Tlatest (e.g., $\mathrm{O}_{1}, \mathrm{O}_{2}$, and $\mathrm{O}_{3}$ in Figure 13(a)), B be the objects located outside of the scope of $K N N$ as Tlatest (e.g., $O_{4}$ and $O_{5}$ in Figure 13(a)), $C$ be the the objects to be included in $K N N$ as Tfuture (e.g., $O_{1}, O_{3}$, and $O_{5}$ in Figure 13(c)), and $D$ be the objects to be located outside of the KNN scope as Tfuture (e.g., $\mathrm{O}_{2}$ and $\mathrm{O}_{4}$ in Figure 13(c)). t_Entity denotes the time range in which all objects included in $A$ can also be included in $C$. Thus, A.D or B.C in which $B$ and $D$ are included are excluded from consideration as a t_Entity target. That is, A.C (e.g., $\mathrm{O}_{1}, \mathrm{O}_{2}, \mathrm{O}_{3}$, and $\mathrm{O}_{5}$ in Figure 13) $\ni t_{-}$Entity, which can be entirely included in the KNN range at Tlatest and Tfuture.

If we assume that the time range of $t_{-}$Entity time is 60 minutes according to Heuristic 1 and that the broadcast cycle is 20 minutes, the client can process $K N N$ by itself, without tuning to the data on the server for up to a maximum of three cycles.

Heuristic 2. (The unchangeable time period in which the order of the target object of $K N N$ does not change). Let $E$ be the set of objects that do not change in the order of KNN from t_Entity to Tfuture. Let $F$ be the set of objects that change in the order of $K N N$ from $t_{-}$Entity to Tfuture (e.g., $O_{1}, O_{2}$ and $O_{3}$ for a period of time $T_{0}-T_{1}$ in Figure 13). Let $F$ be the set of objects that change in the order of $K N N$ from t_Entity to Tfuture. T_Order defines the time range in which all objects that satisfy A.C maintain the same in the order of KNN. That is, it becomes A.C.E $\ni$ T_Order. 
If we assume that the time range of $t_{-}$Entity is 60 minutes for Heuristic 2 and a broadcast cycle of 20 minutes, and for T_Order for a cycle of 20 minutes, the client can return the value stored in the query result queue to the $K \mathrm{NN}$ query result as it is for 20 minutes, without having to listen to the server's data for a maximum of three cycles.

\section{Performance Evaluation}

BBS [29] and DSI [13] are both major indexes, which exploit the wireless data broadcast environment's characteristics for spatial queries. BBS provides excellent AT because it delivers the data sequentially based on the object's location, without separating the index information. On the other hand, DSI is the most widely used index for the selective tuning of clients in a wireless data broadcast environment. Unlike Broadcast Grid Index (BGI) [2] is designed for continuous queries, both DSI and BBS are basically designed for snapshot queries. Moreover, the BGI method is an extended version of the existing DSI method that is suited for continuous spatial queries. Similarly to the previous spatial indexing methods, BGI suffers from the fast-growing index size problem related to the index overlapping pointer. Thus, in this paper, we compare our methods with DSI and BBS. The client's self-processing algorithm is not included in our experimental study. This is because DSI and BBS, the main indexing techniques against which the proposed technique is compared, do not contain the client's self-processing algorithm. Instead of carrying out an experimental study, we defined two heuristic methods (Heuristic 1 and Heuristic 2) regarding the client's self-processing algorithm, and we predicted its performance in the assumed query processing conditions.

\subsection{Performance Analysis}

In this section, we analyze DZI and DSI. DSI uses distributed exponential pointers from each data item. With the exponential pointer, each data object contains pointers that point the IDs of the data items that will subsequently be broadcast. In section 7.1.1 and section 7.1.2, we assume that the average time taken to find a single object in either $K \mathrm{NN}$ queries (i.e., $K=1$ ) or range queries.

\subsubsection{Access Time for DSI and DZI}

Let us compare the access times of the DZI and DSI methods. Because DZI and DSI algorithms have a distributed index structure, the probe wait time can be ignored. We now compare the access time of DSI and DZI.

- DSI: let $e$ be the exponential base $e, N$ be the total number of data, and $f$ be the total index tuple size of the next pointers in each index table ${ }^{5}, h$ be the Hilbert-curve order, $D$ be the size of data, $T_{e 1}$ be the total size of the exponential pointer during a broadcast cycle, where $k=\log _{e} N$ and $I D$ be the object ID. Then $T_{e 1}$ is obtained by:

\footnotetext{
${ }^{5}$ Each index table in DSI has the next pointers that increase exponentially within the range of $N$, the total $r$ amount of data. For example, if $N=1024$, a single index table has 9 next pointers of 1, 2, 4, 8, and up to 1024.
} 


$$
T_{e 1}=\sum_{f=0}^{k} N
$$

Then, the access time for DSI is

$$
A T \_D S I=\frac{T_{e 1}+N \times D+N \times h+N \times I D}{2}
$$

Let $\Re$ be the number of objects that are contained in the grid nodes that belong to the root grid $\mathrm{R}, p^{n}$ be the child node of the root grid node $\mathrm{R}_{n}, z^{n}$ be the number of grid nodes that belong to $\mathrm{R}_{n}, G I D$ be the grid identifier included in $\mathrm{R}_{n}$, and $z^{\prime n}$ be the number of grid-nodes included in the $R_{n}$ (because the number of leaf nodes in $\Re$ is 0 , it is not necessary to indicate it, and therefore, the lowest grid node is excluded from the number). Then, the access time for DZI is

$$
A T \_D Z I=\frac{\sum_{R^{n}=0}^{3} \sum_{p^{n}=0}^{z^{n}} G I D+\sum_{R^{n}=0}^{3} \sum_{p^{n}=0}^{z^{\prime n}} \Re+D \times N}{2}
$$

\subsubsection{Tuning Time for DSI and DZI}

Energy consumption of the indexing techniques can be indirectly compared by measuring how long the client has to stay in active mode to acquire the desired data objects. Measuring the actual energy consumption requires consideration of various factors, such as the size of a single data item and the channel bandwidth. This paper simply uses the client's tuning time as a measure of energy consumption. We now compare the tuning time for DSI and DZI to prove the energy efficiency of our proposed algorithms during spatial query processing. While DSI uses the exponential pointer, which has a redundant structure, our algorithm using DZI allows selective tuning with the ID and the number of objects without the exponential pointer.

Let $N^{\prime}$ be the tuned total number of data and $T_{e 2}$ be the number of indexes from $f^{\prime}$ to $k^{\prime}$, where $k^{\prime}=\log _{e} N^{\prime}$ which the client wakes up and listens to until the desired data are obtained. We obtain:

$$
T_{e 2}=\sum_{f^{\prime}=0}^{k^{\prime}} N^{\prime}
$$

Then, the tuning time (TT) for DSI:

$$
T T \_D S I=h+T_{e 2}+D
$$

Let $L^{n}$ be the number of grid nodes in $\mathrm{R}_{n}$ up to the grid level $n$, until the query processing is completed and $L^{n^{\prime}}$ be the number of grid nodes excluding the leaf grid node 
in the $\mathrm{R}_{n}$ up to the grid level $n$, until the query processing is completed. Let $L^{m}$ be the beginning grid node for tuning, and $L^{m^{\prime}}$ be the beginning grid node for tuning excluding the leaf grid node.

Now, we have:

$$
T T \_D Z I=\sum_{R^{n}=L^{m}}^{L^{n}} \sum_{p^{n}=L^{m}}^{L^{n}} G I D+\sum_{R^{n}=L^{m^{\prime}}}^{L^{n^{\prime}}} \sum_{p^{n}=L^{m^{\prime}}}^{L^{n^{\prime}}} \Re+D
$$

\subsection{Performance Experiments}

Let us now evaluate the efficiency of our algorithms through experimentation. We implemented all methods in $\mathrm{C}++$ and conducted experiments on a machine with a Pentium 3.16 GHz CPU and 3 GByte of RAM. We assume that the client's mobility pattern follows the Random Waypoint Mobility Model [28], which is widely used.

In our experiments, each object begins by pausing for a fixed number of seconds. Then, object selects a random destination in the simulation area and a random speed between 0 and the maximum speed. The object moves to this destination and again pauses for a fixed period before another random location and speed. This behaviour is repeated for the length of the simulation. We now describe the ratio of energy consumption for these states. A mobile client begins by staying in one location for a certain period of time $t$. We assume two energy states: sleep and active mode. In a processor, the doze mode has extremely low power consumption. We assume that the wireless data broadcast channel has a bandwidth of $2 \mathrm{Mbps}$ as the same applied in [2, 29]. We use a uniform dataset (hereafter called $\mathcal{D} 1$ ) with 50,000 points and a real dataset (hereafter called $\mathcal{D} 2$ ) containing 39,231 data objects of MBRs for Montgomery County roads ${ }^{6}$ (see Figures 15(a) and 15(b)). We assign 8 bytes for each pointer and 8 bytes for $2 \mathrm{D}$ coordinates. The default parameter setting in our synthetic data set test is: number of objects $=10,000$, size of data $=256$ bytes, exponential value $e=2$, moving distance $=100 \mathrm{~m}$, the value of $K$ for $K \mathrm{NN}$ search is 3 , and a search range for range query is $300 \mathrm{~m} \times 300 \mathrm{~m}$. Assuming that the data objects are distributed on a $10 \mathrm{~km} \times 10 \mathrm{~km}$ area.

The total exploration time from when the client reads an index at a certain time point after receiving a user's request to obtain the desired final result is assumed as AT. The total amount of time to be awake in order to obtain the desired final result is assumed as TT.

First, we make a comparison between AT for BFD and DFD, according to the increase in the size of the user data for $K \mathrm{NN}$ query. As can be seen in Figure 16, DFD has excellent AT compared to BFD. The user data in BFD is delivered in batches after the index data delivery. Thus, all the index information must be tuned to in order to listen to the user data that will be delivered to the next order. Because the index search method also uses $\mathrm{BFD}$, all index information from the top of the tree must be tuned to first, in order to

\footnotetext{
${ }^{6}$ The dataset available at http://chorochronos.datastories.org/?q=node/17
} 


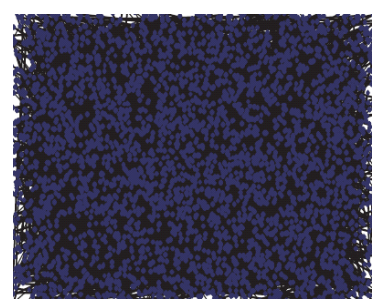

(a)

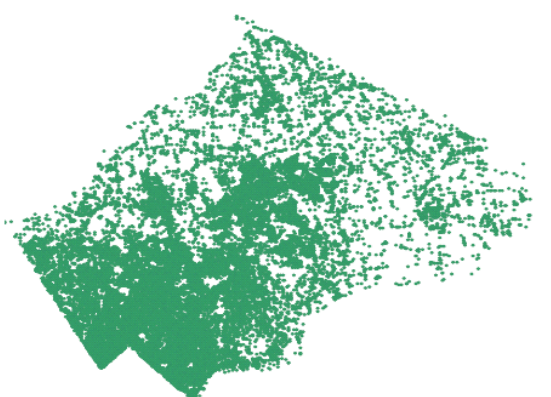

(b)

Figure 15: Datasets for Performance Evaluation. (a) UNIFORM dataset (D1). (b) REAL dataset (D2).

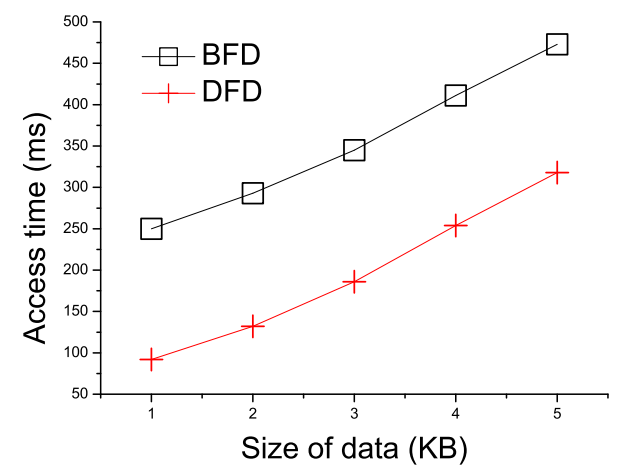

Figure 16: AT for the BFD and the DFD, according to the increase in the size of the user data.

access the sub-index information. The user data is applied to the same process. For this reason, the time consumed in order to obtain the desired information increases according to the increase in the index pointer and data sizes. On the other hand, DFD can obtain the final query result even in the status that involves only listening to the index from the upper part of the tree and the user data. The reason for this is that the index data and the user data are delivered one after another in DFD.

Figure 17(a) shows the results of the measurement of AT according to the increase in $K \mathrm{NN}$. As the $K \mathrm{NN}$ value increases, BFD and DFD must navigate the index data and user data in a broader range. Thus, the search range and AT increase. In particular, we can see that BFD, which can confirm the final query result only after listening to all the index information, shows poor AT results as compared to those of DFD when the $K$ value increases.

Figure 17(b) shows the evaluation of AT in response to an increase in the navigation area of the range query. Similar to the case of $K N N$, in BFD, AT increases sharply, because it must wait until all the index data has been transmitted in order to obtain the user data, and the number of user data required to be confirmed increases as the search region increases. On the other hand, DFD shows far superior AT than BFD, because it is possible to do the query processing via depth-first search, without waiting for the lowest level for no reason. 


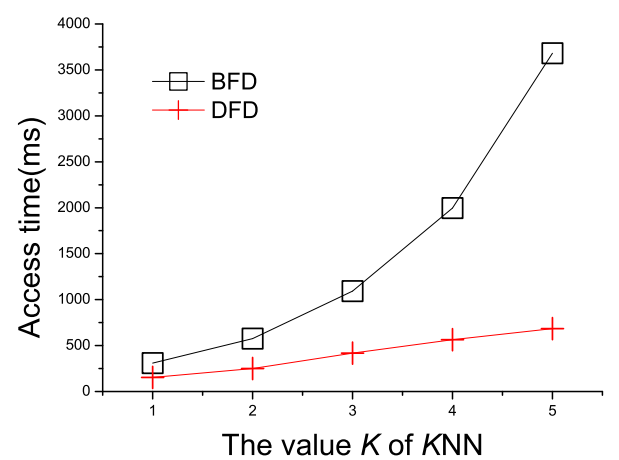

(a)

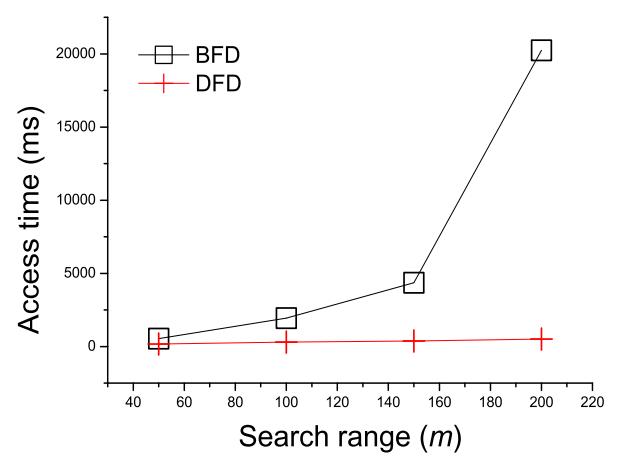

(b)

Figure 17: (a) AT according to the increase in $K N N$. (b) AT in response to an increase in the navigation area of the range query.

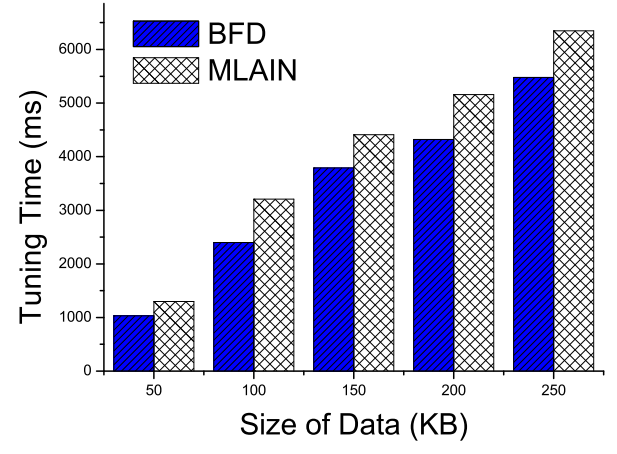

(a)

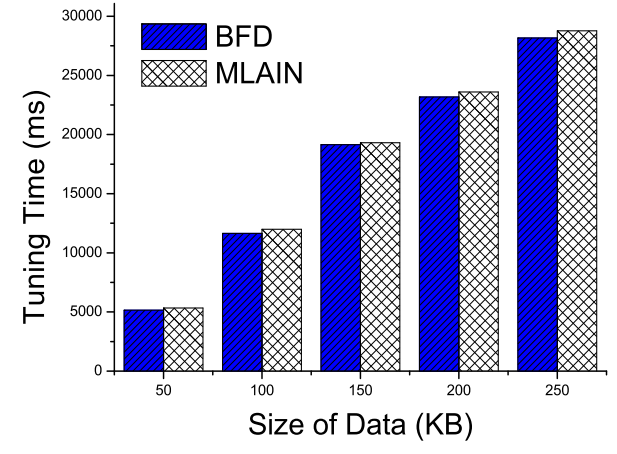

(b)

Figure 18: TT results for the case in which three index techniques are applied to the range query. (a) applying in $\mathcal{D} 1$ (b) applying in $\mathcal{D} 2$.

In the experiment, the energy efficiency was evaluated by comparing the time during which clients stay in active mode. The proposed method enables clients to choose the DFD algorithm to increase search speed or the BFD algorithm to improve energy efficiency. In this experiment, the performance of the proposed method using BFD was compared with that of the MLAIN method. The MLAIN method adopts the depth-first traversal strategy to process a query. In MLAIN, clients stay in active mode while listening to each and every sub-node of a target node Gi. This increases the active mode time and thus decreases the energy efficiency. On the other hand, BFD can enhance energy efficiency via selective listening the nodes that are not necessary for acquiring query results are pruned in advance, at a higher level. Figure 18(a) and 18(b) show the energy efficiency of the proposed and MLAIN methods with regard to data size changes. As can be seen in the figures, the proposed method with BFD has a shorter active mode time than MLAIN, gaining higher energy efficiency. Figure 19(a) and 19(b) present the energy efficiency with search range changes in kNN and range queries. Here, the proposed method also achieves better energy efficiency than MLAIN. 


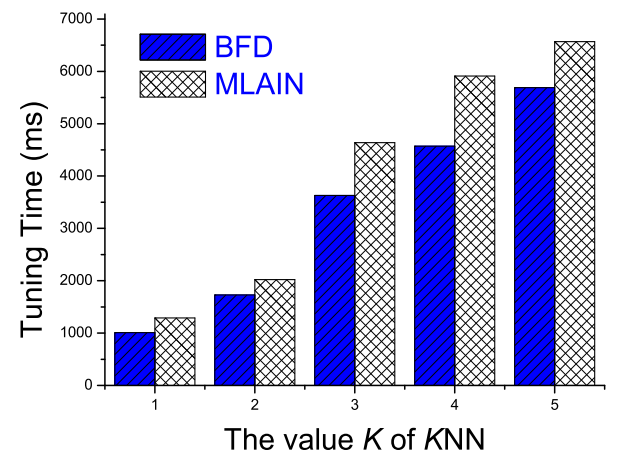

(a)

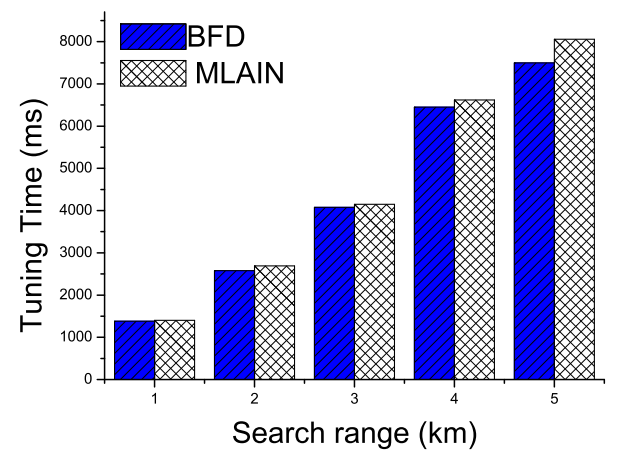

(b)

Figure 19: TT results for the case in which three index techniques are applied to the range query. (a) applying in $\mathcal{D} 1$ (b) applying in $\mathcal{D} 2$.

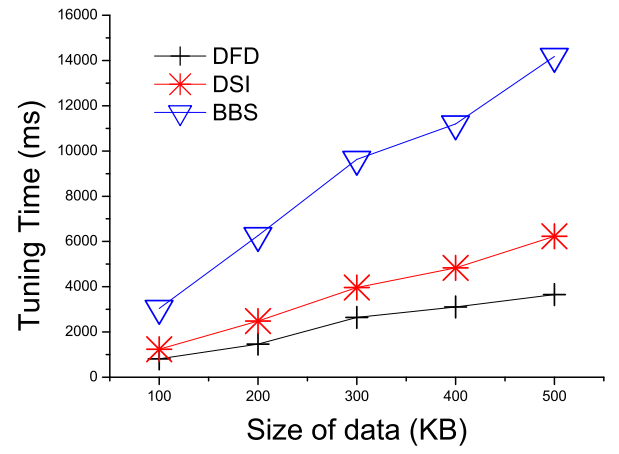

(a)

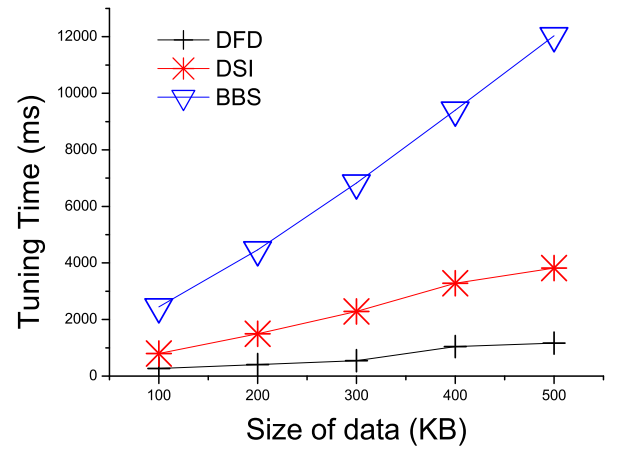

(b)

Figure 20: TT for DFD, DSI, and BBS to KNN queries. (a) applying in $\mathcal{D} 1$ (b) applying in $\mathcal{D} 2$. 


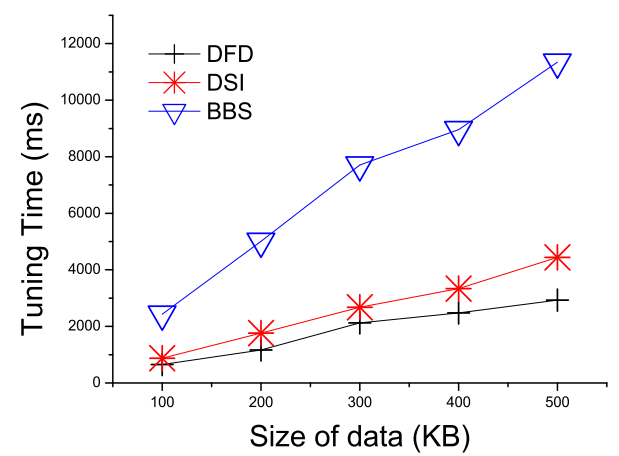

(a)

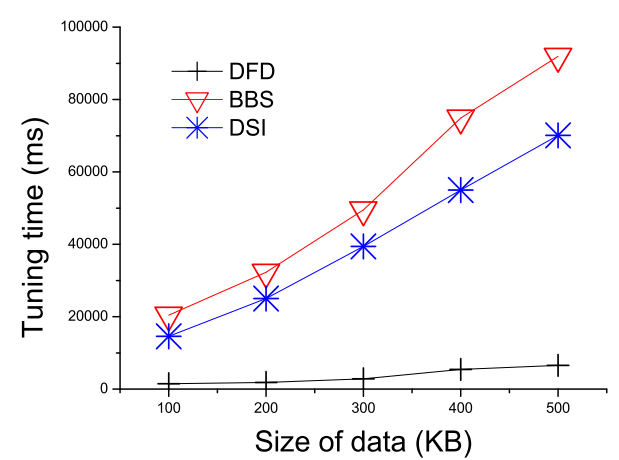

(b)

Figure 21: TT results for the case in which three index techniques are applied to the range query. (a) applying in $\mathcal{D} 1$ (b) applying in $\mathcal{D} 2$.

We compare DFD and DSI according to the increase in data size, and evaluate the performance of $\mathrm{TT}$ and $\mathrm{AT}$ with regard to the BBS techniques. BFD, which displayed relatively poor AT in the previous experiment is excluded from this evaluation. First, TT in $\mathcal{D} 1$ is compared by applying three index techniques to $K \mathrm{NN}$ queries. As can be seen in Figure 20(a), DFD shows quite excellent TT. The reason is that in DSI, a client repeatedly wakes up and falls asleep in the course of the search process on a large number of duplicate pointers, while, in DFD, a client can wait in sleep mode for a longer time because it is possible to listen selectively, using only the identification number of the grid. On the other hand, we find that BBS displays the relatively worst TT, because even though the index size is the smallest because it does not have a pointer that indicates the data location, it does not have a pointer that supports selective tuning, so the time spent in active mode is longer. Figure 20(b) shows the TT results for the three indexes were applied to a $K N N$ query in $\mathcal{D} 2$. We find that DFD shows the relatively best $\mathrm{TT}$ for the same reason as in $\mathcal{D} 1$. On the other hand, we can also see that BBS, which must remain awake for the longest time, displays the poorest TT.

Figures 21(a) and 21(b) show the TT results for the case in which three index techniques are applied to the range query in $\mathcal{D} 1$ and $\mathcal{D} 2$. We find that DFD, also for similar reasons as in the previous experiments, displays quite excellent $\mathrm{TT}$ in the range query.

Next, we compare AT by applying three indexes to a $K N \mathrm{~N}$ query in $\mathcal{D} 1$ and $\mathcal{D} 2$. As can be seen in Figures 22(a) and 22(b), unlike in the previous experimental results, DSI displays the poorest AT, and BBS displays relatively good AT. DSI performs the worst during the time it takes to obtain the final query, due to the burdens of the pointer size and the search cost of a Hilbert-curve. On the other hand, DFD shows excellent AT because its pointer size is relatively small, and it can quickly determine the region not to be listened to, due to the hierarchical tree structure that considers the location. BBS also displays good results compared to the previous TT test, because the burden of the index pointer is on the low end, and it can obtain the final query result without having to listen to all the data. BBS that minimizes the size of the index information reduces the broadcast cycle, which allows for a faster access time in spatial query processing. However, BBS doesn't 


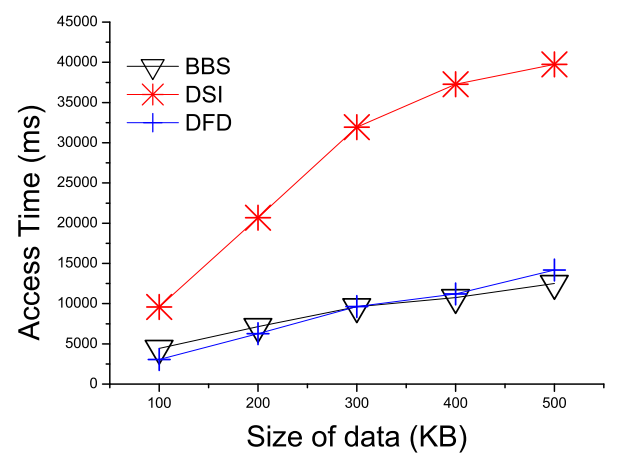

(a)

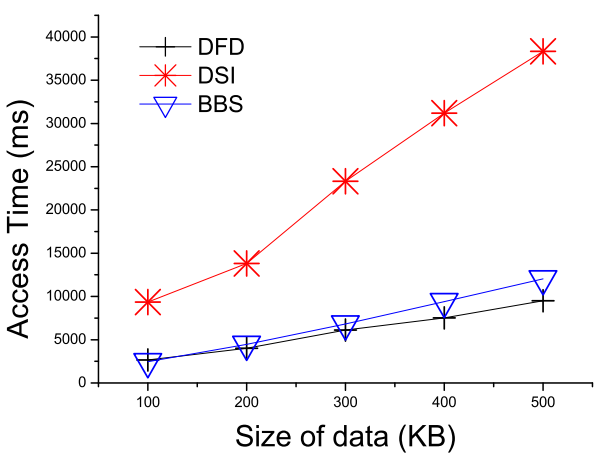

(b)

Figure 22: AT by applying three indexes to a $K N N$ query. (a) applying in $\mathcal{D} 1$ (b) applying in $\mathcal{D} 2$.

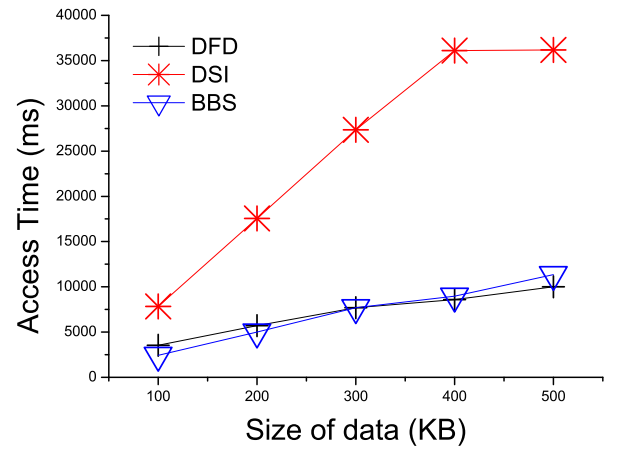

(a)

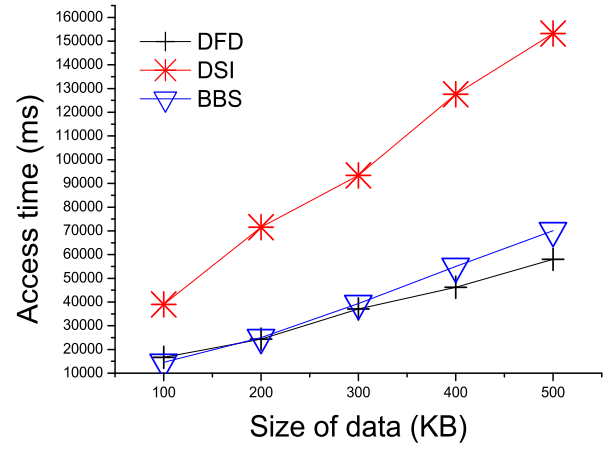

(b)

Figure 23: AT for three index techniques applying to a range query.(a) applying in $\mathcal{D} 1$ (b) applying in $\mathcal{D} 2$.

perform well because of the limitation of selective tuning ability. In general, the client's access time decreases and tuning time increases as the index information decreases.

Figures 23(a) and 23(b) show the results of a comparison between AT in $\mathcal{D} 1$ and $\mathcal{D} 2$, in which three index techniques are applied to a range query. In this case as well, DSI displays the poorest AT, due to the burden of pointer size and the inefficiency of the search method, because the increase in data size increases the number of data that must be listened to.

Next, we perform a test comparing TT according to an increase in $K \mathrm{NN}$ (see Figures 24(a) and 24(b)). As can be seen in Figures 24(a) and 24(b), DFD shows excellent results, because an increase in the $K \mathrm{NN}$ value incurs an increased cost for searching the index data and user data. Figures 25(a) and 25(b) both show the results in $\mathcal{D} 1$ and $\mathcal{D} 2$ for a comparison of $\mathrm{AT}$, in which the value of $K \mathrm{NN}$ is increased from 1 to 5 . As shown in the previous experiment's results, BBS minimizes the index size and improves the access time. DFD shows excellent results, because an increase in the $K \mathrm{NN}$ value incurs an increased cost for searching the index data and user data. Also, there is the least amount of data to be listened to in the active mode, because of the relatively low burden on the index pointer, and the least burden of the mapping calculation of the user data from the index. In BBS, the distribution of objects in a dataset has a direct influence on the data delivery order 


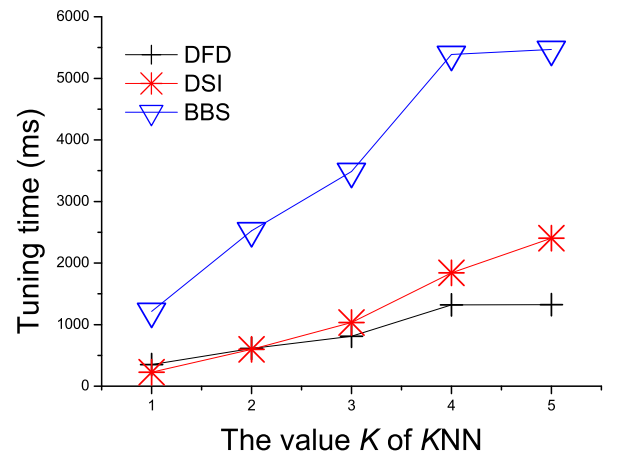

(a)

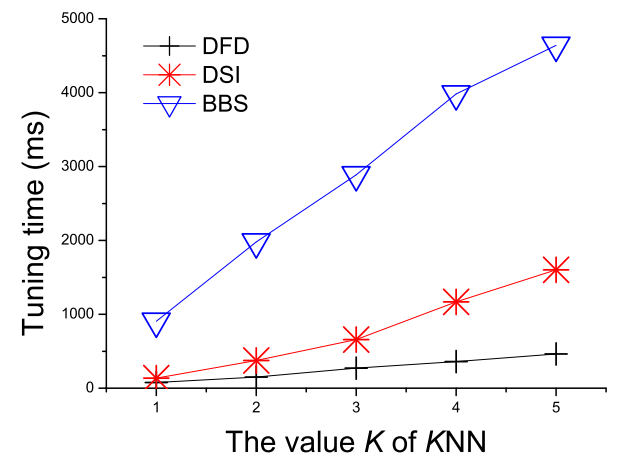

(b)

Figure 24: A comparison according to an increase in $K$ NN. (a) applying in $\mathcal{D} 1$ (b) applying in $\mathcal{D} 2$.

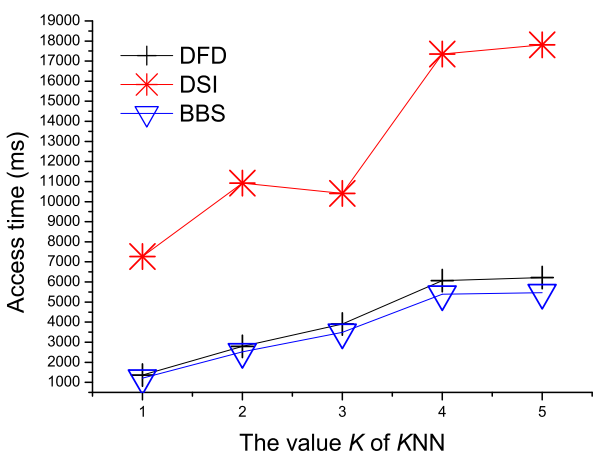

(a)

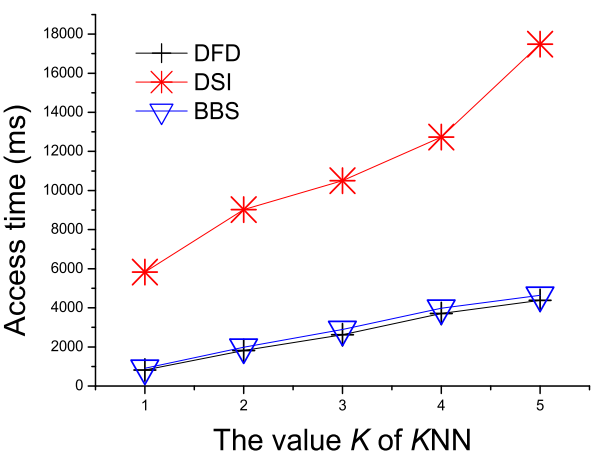

(b)

Figure 25: A comparison of $\mathrm{AT}$, in which the value of $K \mathrm{NN}$ is increased from 1 to 5 . (a) applying in $\mathcal{D} 1$ (b) applying in $\mathcal{D} 2$. 


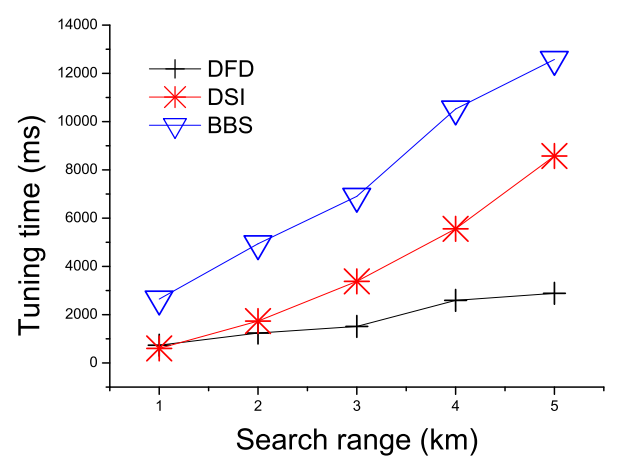

(a)

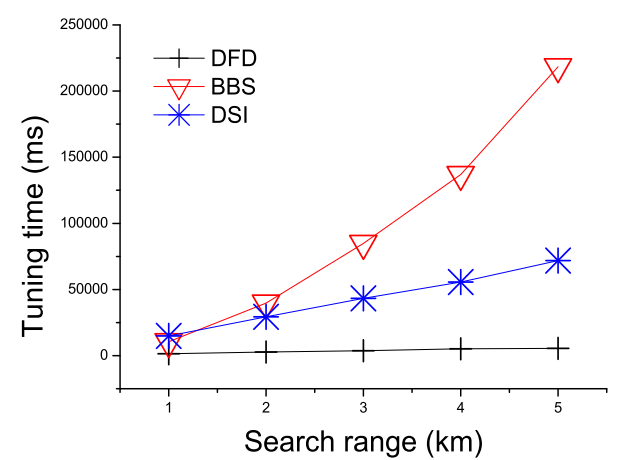

(b)

Figure 26: A comparison for TT in which the search range is increased from $1 \mathrm{~km}$ to $5 \mathrm{~km}$. (a) applying in $\mathcal{D} 1$ (b) applying in $\mathcal{D} 2$.

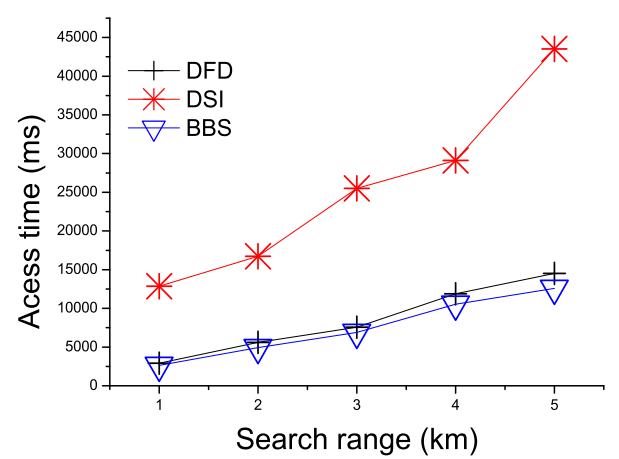

(a)

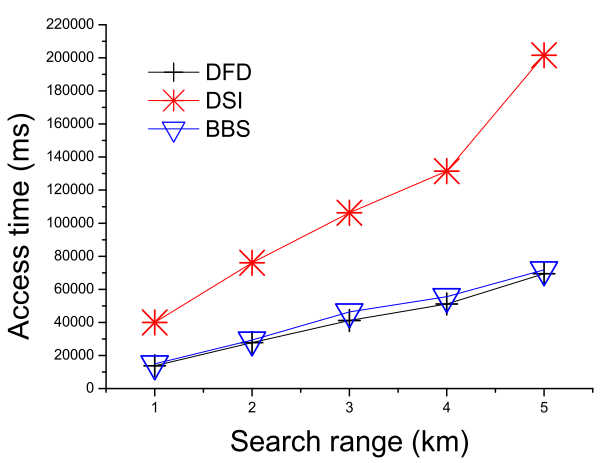

(b)

Figure 27: A comparison according to increases in the search range in the range queries. (a) applying in $\mathcal{D} 1$ (b) applying in $\mathcal{D} 2$.

and search efficiency the objects at the leftmost are delivered first, followed by delivering those at the right-hand side. In the experiment results shown in Figure 25(a), BBS has faster access time than DFD. On the other hand, DFD has a slightly better access time than BBS in Figure 25(b) because of the difference in object distributions.

Figures 26(a) and 26(b) both show the results of a comparison for TT in $\mathcal{D} 1$ and $\mathcal{D} 2$ in which the search range is increased from $1 \mathrm{~km}$ to $5 \mathrm{~km}$. We can see that DFD shows that, as the search range increases, the highest level of performance is also in TT, for the same reason as in the previous test.

Next, we perform a test comparing AT according to increases in the search range in the range queries. Figures $27(\mathrm{a})$ and $27(\mathrm{~b})$ both show the results of a comparison of AT in $\mathcal{D} 1$ and $\mathcal{D} 2$, in which the search range is increased from $1 \mathrm{~km}$ to $5 \mathrm{~km}$. The results show that DSI had the worst result when the search range is increased, for reasons similar to those in the $K \mathrm{NN}$ query processing.

We now compare AT in $\mathcal{D} 1$ in terms of an increase in the size of objects. An increase in the number of objects leads to an increase in the size of the pointer, which represents the 


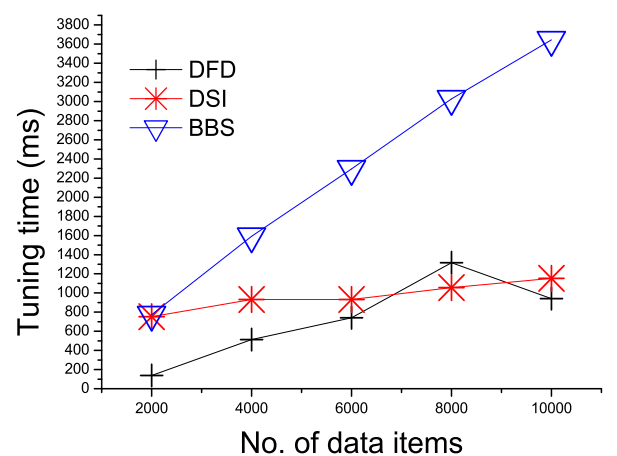

(a)

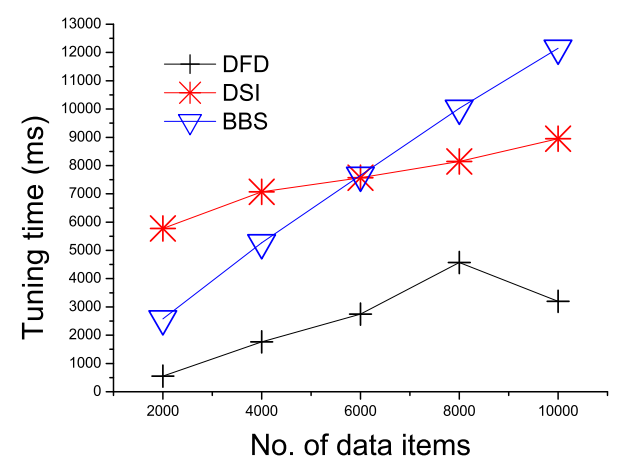

(b)

Figure 28: A comparison and measurement of TT in terms of $K N N$ query. Both (a) and (b) applying in $\mathcal{D} 1$.

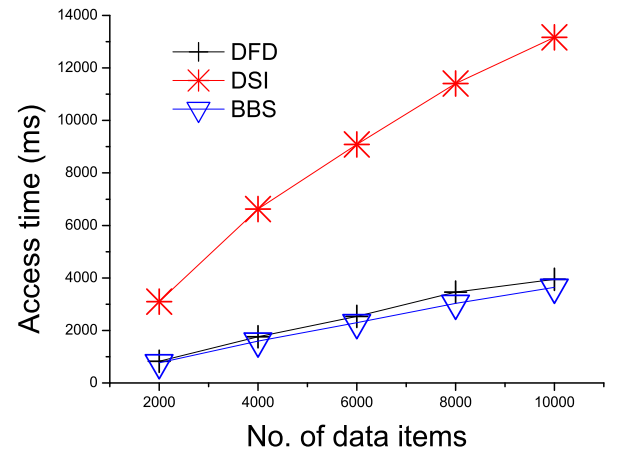

(a)

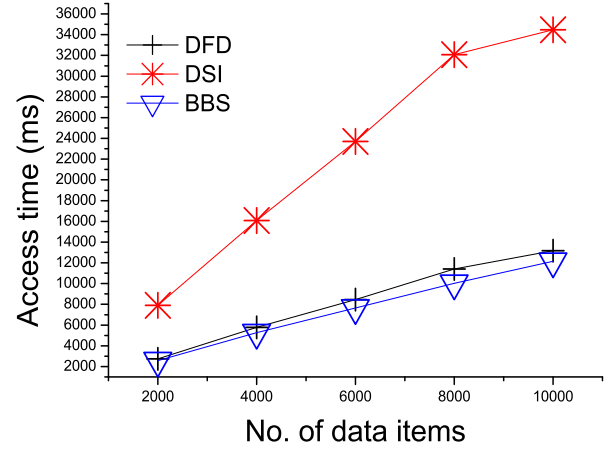

(b)

Figure 29: A comparison and measurement of AT in terms of $K N N$ query. Both (a) and (b) applying in $\mathcal{D} 1$.

object location and delivery time. In the case of DSI, even though a pointer that increases by index $e$ (exponential) is used, the amount of information to be listened to becomes quite large, because the duplicate pointer increases as much as $\log _{e} N$ in each index frame every time the number $N$ of data increases. On the other hand, the listening data and computation time in DZI become relatively small compared to those in DSI, due to the reduction in the cost of duplicate indexing pointers.

Figures 28(a) and 28(b) both show the results for TT as the number of objects increases from 2,000 to 10,000. As can be seen in the test results, DSI displays the worst TT than DFD in the majority of cases, due to the burden of having to read more pointers with the increase in the search range, as well as the problem of having to listen to unnecessary blocks due to the nature of the Hilbert-curve.

Figures 29(a) and 29(b) show the results of a comparison and measurement of AT in $\mathcal{D} 1$ and $\mathcal{D} 2$ in terms of $K \mathrm{NN}$ query, when the number of data is increased from 2,000 to 10,000 . In this case, we can see that DSI shows the worst AT, because it receives a duplicate pointer and forms an inefficient search structure as the number of data increases. On the other hand, we find that DFD, which uses DZI, displays excellent AT, as a result of the lightweight index and fast search results. 
As can be seen in all the experimental results, AT and TT, which are the main elements of performance evaluation, are both affected greatly by the index structure and search method. We find that the search method that uses DZI with DFD shows the best AT and $\mathrm{TT}$ in $K \mathrm{NN}$ and a range query.

\section{Conclusion}

In this paper, we proposed a distribution-based Z-order air index and query processing algorithms, such as BFD (Breadth-First-Delivery), and DFD (Depth-First-Delivery) that reduce AT and TT in a wireless data broadcast environment. In DZI, we are able to reduce the pointer size and search cost, which account for the largest cost in the index structure, by assigning an identification number only to the space of those objects that are actually present. A lightweight index structure reduces the broadcast cycle and index search time, and a hierarchical index structure reduces energy consumption via the client's selective tuning. BFD can improve the efficiency of TT through selective tuning by previewing and pruning the part not to be listened to from the upper node by delivering the DZI tree information from the upper levels to the lower levels. DFD delivers the DZI in depth-first order.

We can improve the performance of AT by determining the final query result by listening to the partial data information, without having to listen to the end node of the tree via the consecutive delivery of index data and user data that belong to the same grid.

Next, we proposed an algorithm that allows the client to self-process a spatial query of a mobile object using the object's location, speed, and direction information without reliance on the server for a period of time. It has proved that DZI with BFD and DFD provides fast response time and minimal energy consumption. In the future, we plan to study the multi-channel broadcast for location-based services.

\section{References}

[1] Bugra G., Aameek S., and Ling L., "Energy efficient exact kNN search in wireless broadcast environments", In Proc. of ACM GIS, pp. 137-146, 2004.

[2] Kyriakos M., Spiridon B., and Dimitris P., "Continuous Monitoring of Spatial Queries in Wireless Broadcast Environments", In IEEE Trans. Mob. Comput., 8(10), pp. 12971311,2009 .

[3] J. Xu, B. Zheng, W.-C. Lee, and D. L. Lee, "Energy Efficient Index for Querying Location-Dependent Data in Mobile Broadcast Environments", In Proc. of the 19th IEEE Int. Conf. on Data Engineering (ICDE '03), pp. 239-250, 2003.

[4] Datta, A., Celik, A., Kim, J.K., VanderMeer, D., and Kumar, V., "Adaptive broadcast protocols to support power conservation retrieval by mobile users", In Proc. of IEEE International Conference Data Engineering (ICDE), pp. 124-133, 1997. 
[5] Datta, A., VanderMeer, D.E., Celik, A., and Kumar, V., "Broadcast protocols to support efficient retrieval from databases by mobile users", ACM Trans. Database Syst., 24(1), pp. 1-79, 1999.

[6] Imielinski, T., Viswanathan, S., and Badrinath, B.R., "Energy efficiency indexing on air", In Proc. of the ACM SIGMOD International Conference on Management of Data, pp. 25-36, 1994.

[7] Imielinski, T., Viswanathan, S., and Badrinath, B.R., "Data on air-organization and access", IEEE Trans. Knowl. Data Eng., 9(3), pp. 353-372, 1997.

[8] Shanmugasundaram, J., Nithrakashyap, A., Sivasankaran, R.M., and Ramamritham, K. "Efficient concurrency control for broadcast environments", In Proc. of ACM SIGMOD International Conference on Management of Data, pp. 85-96, 1999.

[9] Zheng, B. and Lee, D.L., "Information dissemination via wireless broadcast", Commun. ACM, 48(5), pp. 105-110, 2005.

[10] Park, K. and Choo, H., "Energy-efficient data dissemination schemes for nearest neighbor query processing", IEEE Trans. Comput., 56(6), pp. 754-768, 2007.

[11] Park, K. and Valduriez, P., "Energy Efficient Data Access in Mobile P2P Networks", IEEE Trans. Knowl. Data Eng., 23(11), pp. 1619-1634, 2011.

[12] Kyriakos Mouratidis and Man Lung Yiu, "Shortest Path Computation with No Information Leakage", PVLDB, 5(8), pp. 692-703, 2012.

[13] Baihua Z., Wang L., Ken L., Dik L., and Min S., "A distributed spatial index for error-prone wireless data broadcast", $V L D B$ J., 18(4), pp. 959-986, 2009.

[14] Swarup Acharya, Rafael Alonso, Michael J. Franklin, and Stanley B. Zdonik, "Broadcast Disks: Data Management for Asymmetric Communications Environments", In Proc. of SIGMOD Conference, pp. 199-210, 1995.

[15] B. Zheng,W.-C. Lee, and D. L. Lee. "Spatial queries in wireless broadcast systems", Wireless Networks, 10(6), pp. 723-736, 2004.

[16] C. Gotsman and M. Lindenbaum, "On the metric properties of discrete space-filling curves", IEEE Transactions on Image Processing, 5(5), pp. 794-797, 1996.

[17] W.-C. Lee and B. Zheng, "DSI: A Fully Distributed Spatial Index for Location-Based Wireless Broadcast Services", In Proc. of Int'l Conf. Distributed Computing Systems (ICDCS), pp. 349-358, 2005.

[18] Guttman, A., "R-trees: a dynamic index structure for spatial searching", In Proc. of Special Interest Group on Management of Data (SIGMOD), pp. 47-57, 1984. 
[19] Xuan, P., Sen, S., Gonzalez, O., Fernandez, J., and Ramamritham, K., "Broadcast on demand: efficient and timely dissemination of data in mobile environments", In Proc. of IEEE Real Time Technology and Applications Symposium, pp. 38-48, 1997.

[20] Chuan-Ming Liu and Kun-Feng Lin, "Disseminating dependent data in wireless broadcast environments", Distributed and Parallel Databases, 22(1), pp. 1-25, 2007.

[21] P.Nicopolitidis, G.I.Papadimitriou and A.S.Pomportsis, "Exploiting Locality of Demand to Improve the Performance of Wireless Data Broadcasting", IEEE Transactions on Vehicular Technology, vol.55, no.4, pp.1347-1361, 2006.

[22] Zaixin Lu, Yan Shi, Weili Wu, and Bin Fu, "Efficient data retrieval scheduling for multi-channel wireless data broadcast", In Proc. of INFOCOM, pp. 891-899, 2012.

[23] S.E. Hambrusch, C.-M. Liu, W.G. Aref, and S. Prabhakar, "Query Processing in Broadcasted Spatial Index Trees", In Proc. of Int'l Symp. Advances in Spatial and Temporal Databases (SSTD), pp. 502-521, 2001.

[24] Glenn S. Iwerks, Hanan Samet, and Kenneth P. Smith, "Continuous K-Nearest Neighbor Queries for Continuously Moving Points with Updates", In Proc. of VLDB, pp. 512-523, 2003.

[25] G. Ghinita, P. Kalnis and S. Skiadopoulos, "PRIVE: Anonymous Location- Based Queries in Distributed Mobile Systems", In Proc. of International World Wide Web Conference (WWW), pp. 371-380, 2007.

[26] M. F. Mokbel, C.-Y. Chow, and W. G. Aref, "The New Casper: A Privacy-Aware Location-Based Database Server", In Proc. of International Conference on Data Engineering Conference (ICDE), pp. 1499-1500, 2007.

[27] B. Gedik and L. Liu, "A Customizable k-Anonymity Model for Protecting Location Privacy", In Proc. of International Conference on Distributed Computing Systems (ICDCS), pp. 620-629, 2005.

[28] T. Camp, J. Boleng, and V. Davies, "A Survey of Mobility Models for Ad Hoc Network Research", Wireless Comm. and Mobile Computing, vol. 2, no. 5, pp. 483-502, 2002.

[29] Park K., Song M., and Hwang C., "Broadcasting and Prefetching Schemes for Location Dependent Information Services", In Proc. of Int'l Workshop Web and Wireless Geographical Information Systems (W2GIS), pp. 26-37, 2004.

[30] Kyriakos M., "Spatial queries in wireless broadcast environments", In Proc. ACM International Workshop on Data Engineering for Wireless and Mobile Access (MobiDE), pp. 39-44, 2012. 
[31] Y. Xiong, Y. Deng, W. Wang, and J. Ma, "Phoenix: A Collaborative Location-Based Notification System for Mobile Networks", Mathematical Problems in Engineering, Article ID 307498, pp. 12, 2014.

[32] Y. Wang, C. Xu, Y. Gu, M. Chen, and G. Yu, "Spatial query processing in road networks for wireless data broadcast", Wireless Networks (WINET), 19(4), pp. 477494, 2013.

[33] Y. Li, J. Li, L. Shu, Q. Li, G. Li, and F. Yang, "Searching continuous nearest neighbors in road networks on the air", Information Systems (IS), 42, pp. 177-194, 2014.

[34] W. Sun, C. Chen, B. Zheng, C. Chen, and P. Liu, "An Air Index for Spatial Query Processing in Road Networks", IEEE Trans. Knowl. Data Eng (TKDE), 27(2), pp. 382-395, 2015.

[35] K. Park and P. Valduriez, "A Hierarchical Grid Index (HGI), spatial queries in wireless data broadcasting", Distributed and Parallel Databases (DAPD), 31(3), pp. 413-446, 2013.

[36] P. Nagarkar, K. S. Candan, and A. Bhat, "Compressed Spatial Hierarchical Bitmap (cSHB) Indexes for Efficiently Processing Spatial Range Query Workloads", In Proceedings of the VLDB Endowment (PVLDB), 8(12), pp. 1382-1393, 2015.

[37] Y. Li, L. Shu, R. Zhu, and L. Li, "A novel distributed air index for efficient spatial query processing in road sensor networks on the air", Int. J. Communication Systems 30(5), pp. 1-23, 2017.

[38] J. Shen and M. Jian, "Spatial query processing for skewed access patterns in nonuniform wireless data broadcast environments", International Journal of Ad Hoc and Ubiquitous Computing, 25(1/2), pp. 4-16, 2017.

[39] D. Song and K. Park, "A partial index for distributed broadcasting in wireless mobile networks", Inf. Sci. 348, pp. 142-152, 2016.

[40] Y. Li, G. Li, J. Li, and K. Yao, "SKQAI: A novel air index for spatial keyword query processing in road networks. Inf. Sci, 430, pp. 17-38, 2018.

[41] M. Veeresha and M. Sugumaran, "Continuous k-Nearest Neighbor Queries in Road Networks", In Proc. of International Conference on Inventive Systems and Control, pp. 1-4, 2017.

[42] S. Im, and J. Choi, "MLAIN: multi-leveled air indexing scheme in non-flat wireless data broadcast for efficient window query processing", Computers and Mathematics with Applications, 64(5), pp. 1242-1251, 2012. 